\title{
Movimientos sacádicos como un indicador en la veracidad del testimonio: Una revisión sistemática
}

\section{Saccadic movements as an indicator in the veracity of testimony: A systematic review}

DOI: $10.46932 /$ sfjdv2n2-064

Received in: March 1st, 2021

Accepted in: May 30th, 2021

\section{Walter Jacinto Portoviejo Fernández}

Tercer nivel en Psicología clínica.

Institución: Unidad de Posgrados de la Universidad Católica de Cuenca.

Dirección laboral: Ecuador, Azuay, Gualaceo, Luis Ríos Rodríguez y Antonio Vera.

Correo electrónico: walterportoviejo@yahoo.com

\section{Geovanny Genaro Reivan Ortiz}

$\mathrm{PhD}$ en Psicología mención "metodología y estadística"

Institución: Laboratorio de Psicología Básica, análisis conductual y desarrollo programático.

Universidad Católica de Cuenca, Campus CIITT.

Dirección personal: Ecuador, Azuay, Cuenca, parroquia Ricaurte, Vía Ricaurte Bibín frente a Plásticos Rival.

Correo electrónico: greivano@ucacue.edu.ec greiva@palermo.edu

\section{Pedro Carlos Martínez Suarez \\ $\mathrm{PhD}$ en Psicología.}

Institución: Laboratorio de Psicometría, etología y Psicología comparada. Universidad Católica de

Cuenca, Campus CIITT.

Dirección personal: Ecuador, Azuay, Cuenca, parroquia Ricaurte, Vía Ricaurte Bibín frente a Plásticos Rival.

Correo electrónico: pmartinezs@ucacue.edu.ec

\section{RESUMEN}

Este trabajo de revisión sistemática tiene como objetivo principal el sintetizar los artículos de investigación de alto impacto que contengan información relacionada a los movimientos sacádicos y sus componentes como indicadores del engaño, para ello se utiliza un diseño de revisión documental en donde se implementa el método Prisma para contar con criterios de inclusión y exclusión de las investigaciones a utilizar; después de realizar la búsqueda por las diferentes revistas científicas se obtienen al final 38 artículos los cuales cumple con los criterios de selección. Al realizar el análisis de los trabajos de investigación para la variable de carga cognitiva se obtiene 9 artículos que confirman, 7 que niegan y 4 mencionan que los datos no son concluyentes, en cuanto a la siguiente variable que es la dilatación pupilar la situación cambia ya que 12 artículos confirman que es posible detectar la mentira mediante la medida de la pupila y solo 1 menciona que los resultados no son concluyentes, en cuanto a la última variable los resultados cambian de nuevo ya que al terminar de revisar las investigaciones se pudo obtener 7 artículos que confirman el postulado y 4 que lo niegan dejando 3 artículos que mencionan que los resultados no son concluyentes. Al contar con la información necesaria se concluye que las variables movimientos sacádicos y carga cognitiva no muestran evidencia suficiente para considerar que pudieran funcionar como indicadores en la veracidad del testimonio, sin embargo, la variable dilatación pupilar muestra mejores evidencias para cumplir con el trabajo de indicador de la verdad. 
Palabras clave: Mentira, carga cognitiva, dilatación pupilar, movimiento de los ojos.

\begin{abstract}
The main objective of this systematic review is to synthesize high impact research articles containing information related to saccadic movements and their components as indicators of deception, for this purpose a documentary review design is used where the Prisma method is implemented to have inclusion and exclusion criteria of the research to be used; after performing the search through the different scientific journals, 38 articles are obtained which meet the selection criteria. When analyzing the research works for the cognitive load variable, 9 articles confirm, 7 deny and 4 mention that the data are not conclusive. As for the next variable, which is pupil dilation, the situation changes since 12 articles confirm that it is possible to detect lying by measuring the pupil and only 1 mentions that the results are not conclusive, As for the last variable, the results change again, since after reviewing the research, 7 articles confirm the postulate and 4 deny it, leaving 3 articles that mention that the results are not conclusive. Having the necessary information, it is concluded that the variables saccadic movements and cognitive load do not show sufficient evidence to consider that they could function as indicators of the truthfulness of the testimony; however, the variable pupillary dilation shows better evidence to fulfill the job of truth indicator.
\end{abstract}

Keywords: Lying, cognitive load, pupillary dilation, eye movement.

\title{
1 INTRODUCCIÓN
}

Desde algunos años atrás se viene intentando descubrir una manera eficaz y eficiente para la identificación de la verdad y la mentira dentro en un discurso, con el avance de las tecnologías y el trabajo de investigaciones en este campo los autores han conectado a los movimientos sacádicos, carga cognitiva, y dilatación pupilar como indicadores de veracidad dentro del testimonio, por otro lado, las investigaciones recientes como las de: Jeffrey et al. 2018, Curci et al. 2018 y Blandón et al. 2017, muestran datos contradictorios a las afirmaciones anteriores, debido a esto, surge la necesidad realizar un trabajo de revisión sistemática para llegar a una conclusión que permita aclarar la duda surgida dentro de esta área de la investigación.

El estudio de la mentira inicia con Leonardo Keeler (1938), quien inventa el polígrafo como medio para descubrir mentiras dentro del testimonio de personas acusadas de crímenes, desde entonces las investigaciones han evolucionado tomando nuevas variables que intentan cumplir con el papel de indicadores de la veracidad al momento de analizar los discursos, sin embargo, y a pesar del surgimiento de nuevas variables los resultados no han mejorado ya que la investigación de Leal y Vrij (2010), donde exponen resultados similares entre parpadeos de personas que dicen la verdad y de las que está diciendo mentiras y con esos resultados en mente se fortaleció la idea de llevar acabo esta revisión sistemática para intentar aclara estos conflictos entre resultados.

La detección de la mentira en el ámbito de la seguridad siempre ha sido un problema ya que las autoridades tienen que discernir entre la verdad y la mentira justo en el momento de la declaración del acusado, por lo que surge la necesidad de una ayuda a la justicia al momento de llegar a una condena o 
exculparlo. Se justifica lo antes mencionado con la investigación de Vrij et al. (2008), con miembros de la policía que logra identificar la existencia de dificultades para detectar la veracidad del diálogo de los acusados, por esta razón se han inventado una variedad de técnicas con poca eficacia al momento de aplicarlas en los sospechosos, tanto en el campo o como en ambientes controlados.

El artículo de revisión sistemática tiene como propósito sintetizar los artículos de investigación que contengan información relacionada a los movimientos sacádicos, los cuales son conocidos como indicadores para determinar la veracidad del discurso, a través de la revisión sistemática de artículos empíricos de cronología amplia, para identificar los componentes que conforman los movimientos sacádicos, conocer la métrica de los componentes en los movimientos sacádicos, e identificar la relación de los movimientos sacádicos con el nivel de carga cognitiva.

Por lo tanto, el estudio tiene como objetivo sintetizar la información relacionada a los movimientos sacádicos durante la determinación de la veracidad del discurso, mediante de la revisión sistemática de artículos empíricos de cronología amplia, para identificar los componentes que conforman los movimientos sacádicos, conocer la métrica de los componentes, e identificar la relación de los movimientos sacádicos con el nivel de carga cognitiva y sobre todo identificar si son útiles como indicadores específicos.

El análisis de datos realizadas dejó ver resultados variados y algunos datos variables que se intenta responder a qui, como la idea que, los movimientos sacádicos sirven de indicadores ante la verificación del discurso, o también que los movimientos sacádicos están regidos únicamente por la carga cognitiva y, por último, intentaremos demostrar si existe evidencia que relacione a la carga cognitiva con la veracidad de un discurso.

\section{MÉTODO}

Diseño: revisión documental - revisión sistemática

\section{Diagrama Prisma:}




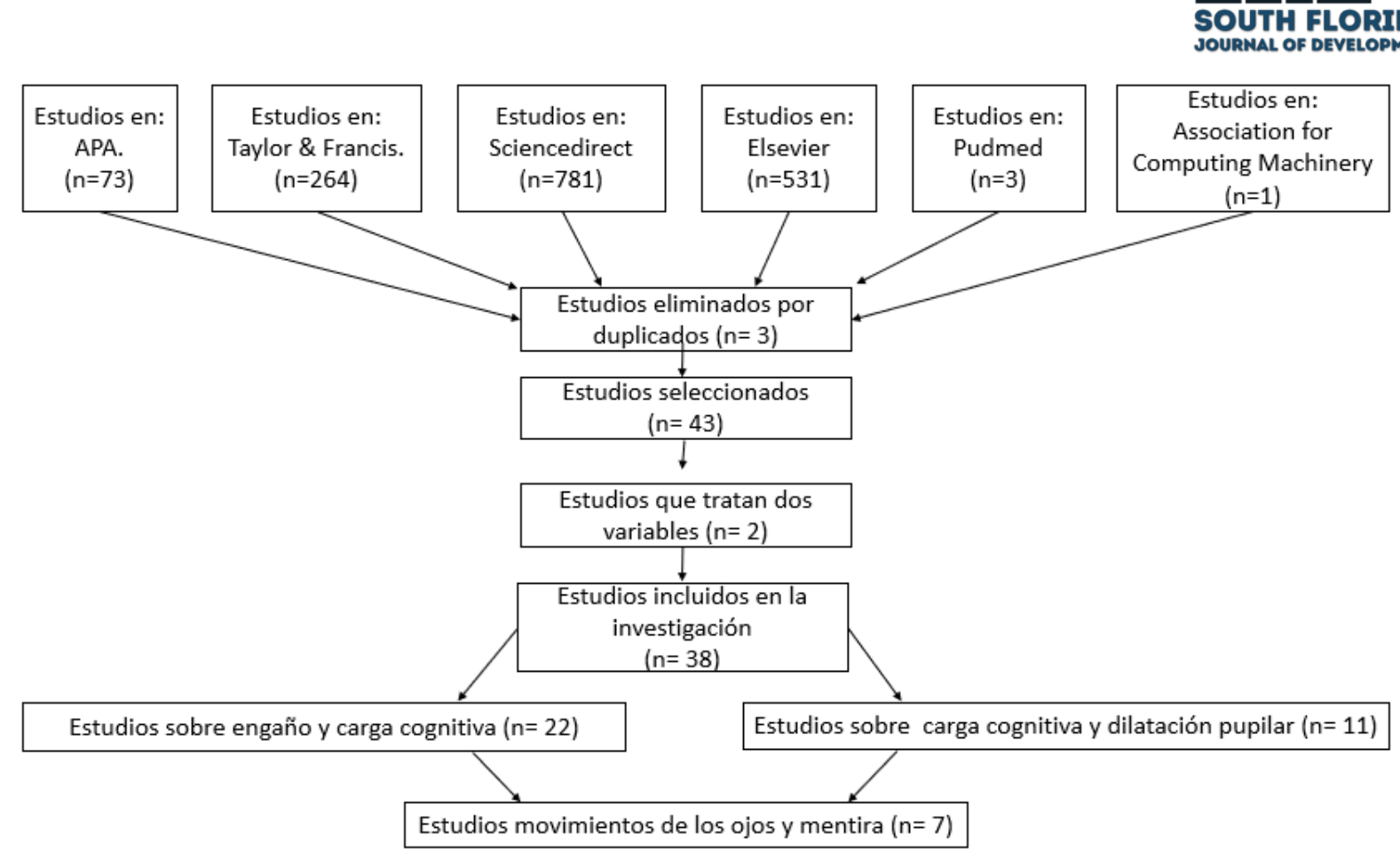

\subsection{PROTOCOLO}

Se realizó mediante la calidad de la presentación de informes según las directrices PRISMA (presentación de informes preferidos para revisión sistemáticas y metaanálisis) y una lista de verificación AMSTAR (herramienta para evaluar la calidad de la revisión bibliográfica; Shea, 2007). Se siguió los procedimientos descritos para el desarrollo de Revisiones Sistemáticas previstas por el Manual Cochrane (Higgins \& Green, 2011).

\subsection{CRITERIOS DE SELECCIÓN}

Para la validación de la información se buscó artículos empíricos en inglés y que sean de corte transversal y longitudinal, además, que correspondan a las bases de datos bibliográficos: ELSEVIER, PubMed, Taylor \& Francis, Wiley y Springer.

Entre otros criterios de inclusión, tenemos a los artículos que utilizaron unidades de medida fiables y válidas para la variable evaluada, también artículos con una muestra poblacional adulta > 18 (humanos), en cuanto a los criterios de exclusión podemos decir que no ingresaran los artículos que correspondan una población infantil y estudios que no se determinen como: estudios de caso, revisiones literarias guiadas, resúmenes y conferencias, cartas al editor y fuente de base de datos grizzy. 


\subsection{IDENTIFICACIÓN Y SELECCIÓN DE ESTUDIOS}

Se realizó una búsqueda avanzada con metodología de comando tesauro (AND, OR, +, -, ALLINTITLE, ALLINTEXT, FILETYPEII) de literata disponible en las bases de datos científicas con las palabras clave: "lie”, “deception", "movement eyes" "cognitive"

Cada artículo fue evaluado con los criterios de selección a través del título, abstract y palabras clave de cronología libre. La búsqueda inicial se realizó el 19 de septiembre de 2020 para identificar los artículos que cumplieran con los criterios ya señalados.

La selección de artículos fue realizada por el autor principal de forma independiente, por lo que, al realizar esta selección no se contaba con los criterios claros generaron una inseguridad en la elección de los artículos y para superar este inconveniente se determinó la discrepancia médiate un informe.

\subsection{EXTRACCIÓN DE DATOS}

Se utilizaron procesos uniformes en la extracción de datos de los estudios incluidos. De cada estudio se extrajo la información general relacionada con las características de estudio por una tabla informativa (base de datos, autor, año, título, objetivo, método, resultado, aspecto discutido) para la elaboración de un diagrama de flujo para determinar el contraste de totalidad del estudio.

\subsection{EVALUACIÓN DE LA CALIDAD METODOLÓGICA DE LOS ESTUDIOS}

Se utilizó el check-list NICE con una escala de calificación integral para evaluar la composición de los artículos empíricos (análisis metodológico, procedimental y estadístico).

\section{RESULTADOS:}

\subsection{COGNICIÓN Y EL ENGAÑO}

Carga cognitiva, es entendida por O’Donnell \& Eggemeier (1986), en Cárdenas, (2018, p.44), como: "la cantidad de recursos cognitivos que una persona necesita para realizar una tarea determinada".

En una investigación realizada en oficiales de policías por Man y Vrij. (2007), descartan la idea que, para detectar mentiras de manera eficiente en personas sospechosas de algún tipo de delito, no se tiene que centrarse en que la persona está tensa o no, sino más bien dirigen su atención para detectar sin están pensando demasiado o realizan intentos por controlar su comportamiento, de esta manera asegura el autor que los miembros de la policía son capaces de determinar la veracidad del discurso en personas sospechosas de actos delictivos.

Una de las técnicas utilizadas dentro del experimento realizado por Vrij et al. (2008), es pedir a los participantes que realice un recuento de los hechos empezando desde el final de la historia hasta el 
inicio, con los resultados de esta investigación se logra demostrar que, al esforzar al cerebro para que organice la información de manera inversa aparece una saturación en la carga cognitiva con la que se vuelve más fácil detectar los indicios relacionados con la mentira dentro del discurso.

Dukala et al. (2008), en su trabajo de investigación a presos con delitos sexuales pudo comprender que muchos agresores al mentir creen firmemente que lo que dicen es verdad, y esto se debe a las distorsiones cognitivas que se van formado a lo largo de la vida, incluso muchos de estos agresores creen que sus cogniciones son normales por lo que al testificar según ellos están diciendo la verdad, por lo tanto, detectar la mentira en este tipo de personas se ha vuelto una tarea muy complicada.

Muestra una mejor fiabilidad un detector de mentiras que se basa en el comportamiento que en la cognición ya que estos recogen y procesan información limitada, por otro lado, podemos decir que muestran un mejor nivel de fiabilidad los detectores basados en el comportamiento, esto no quiere decir que sean completamente fiables ya que estos basan su análisis en la comunicación no verbal, y al ponerlo en frente de una persona que se encuentra entrenada y con el conocimiento necesario para controlar el lenguaje no verbal su fiabilidad cae por completo. (Hugh y Young. 2009)

La necesidad de encontrar herramientas eficaces dentro del ámbito forense ha incentivado a experimentar con varias técnicas, la más prometedora dentro de esta investigación es la entrevista cognitiva, permitiendo llegar a Bembibre \& Higueras (2011) a la conclusión que muestra una eficiencia global significativa para la detección de mentiras durante la declaración de presuntos delincuentes.

Con el trabajo de Bembibre \& Higueras (2012), se determinó que las personas cuando realizan una mentira por otra persona que consideran importante para ella e intentan exculpar de conductas negativas a las mismas, se genera una mayor carga cognitiva y menos procesamiento de la información sensorial, temporal y contextual, de la misma manera, se observa resultados similares en el procesamiento de la información sensorial y cognitiva cuando se miente para a culpar a una persona.

Como explica Cook et al. (2012), dentro de la mentira intervienen múltiples factores, por lo cual, se vio la necesidad de investigar y determinar cuáles son los predominantes, para dicha tarea se realizó una investigación donde se puedo concluir que son dos los procesos cognitivos que intervienen de manera directa en el engaño y a dichos procesos son: la vigilia y la estrategia los cuales permiten estar atentos a no cometer errores de secuencia y lógica dentro del discurso.

Con el fin de contribuir con el estudio de la relación entre mentira y carga cognitiva Bockstaele et al. (2012), realizan un estudio con 42 personas en las que pudo llegar a dos conclusiones, en la primera; los resultados afirman que para mentira de mejor manera es necesario pasar por un entrenamiento, el cual no necesariamente tiene que ser estricto, más bien por una etapa de planificación y repaso. En cuanto a la segunda conclusión el autor menciona que los resultados salieron contrarios ya que en el ensayo donde se 
les entreno a las personas para decir la verdad, no se observó ninguna alteración en la carga cognitiva, indicando que la mentira se vuelve inidentificable si una persona se prepara o tiene practica en mentira ya que su carga cognitiva no se ve alterada.

Walczyk et al. (2013), realizo una revisión crítica de las técnicas para la detección de la mentira basadas en la carga cognitiva, una de las técnicas más controversiales y las que critico son las preguntas controladas basadas en el polígrafo las que fueron criticadas por el consejo nacional de investigación (2003), en donde afirman que estas pruebas carecen de teoría valida, por lo que el autor sugiere que al momento de proponer un modelo teórico se lo haga con una evidencia científica.

Los estudios realizados por Pfister et al. (2014), mediante el uso de electroencefalograma se confirmó que las mentiras demandan alto nivel de carga cognitiva, sin embargo, este estudio no fue concluyente por lo que el autor llega a la conclusión que para lograr una detección de la mentira de manera eficiente es necesaria más investigaciones ya que en la actualidad sigue siendo esquiva.

Terola et al. (2014), propuso realizar la siguiente investigación desde un punto de vista forense para evaluar el grado de alteración en la carga cognitiva en el momento de identificar a las personas que dicen la verdad o miente, obteniendo los siguientes resultados en un rango de $7 \%$ de ciertos para las personas que se encuentra diciendo mentiras, mientras que el $31 \%$ para la tasa de aciertos en las personas que se encuentra diciendo la verdad, estos resultados llevaron a discutir entre los autores y a determinar que este tipo de pruebas son inexactas y proponen la necesidad de seguir investigando en esta área si se espera conseguir resultados más fiables.

Para determinar la validez de la detección de la mentira en entrevistas colectivas, Vernham \& Vrij. (2015), realizan una revisión sistémica donde establece que las señales comunicativas e interactivas, tales como: realizar preguntas, terminar las frases, corregirse, mirarse e interrumpirse son claros indicadores de engaños, estos puntos son el resultado de entrevista dirigidas a la función de la memoria, por lo que sugiere que es posible determinar la veracidad del discurso si se aumenta la carga cognitiva.

Con el fin de seguir estudiando el campo de la mentira, Hu et al. (2015), en su trabajo de investigación desarrolla el novedoso software llamado "sígueme", donde estudiantes de pregrado fueron instruidos para mentira durante una entrevista en la que afirmaban estar cursando un posgrado, los resultados permitieron llegar a la conclusión que decir una mentira descarada requiere más esfuerzo cognitivo que decir la verdad.

Volvemos a hablar de la técnica mas utilizada dentro del ámbito de la justicia que es la entrevista cognitiva, a hora Louge et al. (2015) señala que en la investigación realizada se puede establecer una diferencia significativa en cuando a la detención de mentiras y verdades, además, establece que para la correcta evaluación de la veracidad es necesario llevar una evaluación afectiva. 
Durante el año 2016 Elliott, y Leach, realizan una investigación centrada en el engaño en personas nativas de 4 niveles de habla inglesa, donde se les observo para intentar detectar el engaño en cada discurso de las personas, se llegó a la conclusión que las personas con nivel más bajo del manejo del lenguaje inglés realizan más esfuerzo cognitivo por lo tanto se logra discriminar de mejor manera la verdad o la mentira dentro del dialogo.

Dentro de los experimentos que trabajan con las variables de mentira y carga cognitiva se puede encontrar a los autores Foerster et al. (2017); quienes realizan dos experimentos que ayudan a llegar a la conclusión de que las falsas cuartadas pueden eliminar a las acciones realizadas, por lo tanto, nos sugiere que las técnicas de enfoque cognitivo para la detención de las mentiras se vuelven limitada.

Un meta-análisis realizado por Suchotzki et al. (2017), con el fin de llegar una conclusión de la efectividad de las técnicas cognitivas para la detección de la mentira, en esta investigación se explica que hasta el momento y con la información obtenida de los estudios no se puede confirmar con certeza que la carga cognitiva tenga una relación directa con la mentira.

Existen muchos trabajos de investigación que se centran en determinar la carga cognitiva como un indicador del engaño, para lo cual se realizó una revisión sistemática a cargo de Blandón et al. (2017), donde llegaron a la conclusión que la carga cognitiva no puede funcionar como indicador en las mentiras ya que se ve afectada por las emociones, además concuerdan que mentir no siempre es más complejo que decir la verdad.

Las investigaciones para detectar las mentiras se realizan por todo el mundo, Frank et al. (2018), realiza un experimento con personas nativas de Holanda, donde logró determinar que al usar un idioma extranjero se genera una actividad cognitiva elevada que podía reducir notablemente las diferencias entre mentiras y verdades, además, se observó que las personas luchan por recuperar rápidamente la verdad en otro idioma.

Entre las investigaciones para detector las mentiras en pacientes que manifiestan dolores psicológicos, físicos o en el área forense con fines lucrativos, Walczyk et al. (2018), Realiza un análisis de múltiples métodos que están destinados a detectar la veracidad del discurso a través de la carga cognitiva, logran observar la existencia de un alto índice de falsos positivos y falsos negativos en todas las pruebas volviéndose imposible determinar cuál es la técnica que mejor investiga la veracidad del discurso.

Las investigaciones para detectar la mentira se van dando por todas las áreas y campos, por lo que Curci et al. (2018), indago en el área jurídica sobre todo la capacidad para detectar mentiras de los jueces, el resultado de esta investigación permitió conocer las premisas que usan para identificar mentiras, las técnicas de los jueces era medianamente precisa y se asociaba al sentido de la confianza individual, 
además se logra conocer que las variables que usan para la identificación de las mentiras son las características emocionales, teniendo en cuenta la complejidad cognitiva y la escasez de manifestaciones expresivas.

Como mencionan Aviad et al. (2019), en la investigación que intenta establecer una relación entre la carga cognitiva y la mentira, los resultados que obtiene son importantes ya que se observa un aumento en la actividad cognitiva al momento de decir una mentira, sin embargo, estos resultados se ven cuestionados por el conflicto que se aprecia al momento de ejecutar respuestas automatizadas de verdad o mentira.

CARGA COGNITIVA Y ENGAÑO.

Tabla 1.

\begin{tabular}{|c|c|c|c|}
\hline NOMBRE DEL ARTICULO. & CONFIRMA. & NIEGA. & $\begin{array}{l}\text { NO } \\
\text { CONCLUYENTE }\end{array}$ \\
\hline $\begin{array}{l}\text { Police officers' judgements of veracity, tenseness, } \\
\text { cognitive load and attempted behavioural control in real- } \\
\text { life police interviews. Man y Vrij. (2007) }\end{array}$ & $\mathrm{X}$ & & \\
\hline $\begin{array}{l}\text { Increasing cognitive load to facilitate lie detection: The } \\
\text { benefit of recalling an event in reverse order.Vrij et al. } \\
(2008)\end{array}$ & $\mathrm{X}$ & & \\
\hline $\begin{array}{l}\text { Detecting deception: does the cognitive interview impair } \\
\text { discrimination with CBCA criteria in elderly witnesses?. } \\
\text { Dukala et al. (2008) }\end{array}$ & & $X$ & \\
\hline $\begin{array}{l}\text { Self-reported cues about deceptive and truthful } \\
\text { communication: The effects of cognitive capacity and } \\
\text { communicator veracity. } \\
\text { Hugh y Young (2009) }\end{array}$ & & $\mathrm{X}$ & \\
\hline $\begin{array}{l}\text { Differential effectiveness of the cognitive interview in a } \\
\text { simulation of testimony. Bembibre \& Higueras (2011) }\end{array}$ & $\mathrm{X}$ & & \\
\hline $\begin{array}{l}\text { Comparative analysis of true or false statements with the } \\
\text { source monitoring model and the cognitive interview: } \\
\text { special features of the false accusation of innocent people. } \\
\text { Bembibre \& Higueras (2012) }\end{array}$ & $\mathrm{X}$ & & \\
\hline $\begin{array}{l}\text { Lyin' eyes: Ocular-motor measures of reading reveal } \\
\text { deception. Cook et al. (2012) }\end{array}$ & $\mathrm{X}$ & & \\
\hline $\begin{array}{l}\text { Learning to lie: effects of practice on the cognitive cost of } \\
\text { lying. Bockstaele et al. (2012) }\end{array}$ & & $\mathrm{X}$ & \\
\hline $\begin{array}{l}\text { Advancing lie detection by inducing cognitive load on } \\
\text { liars: a review of relevant theories and techniques guided } \\
\text { by lessons from polygraph-based approaches. Walczyk et } \\
\text { al. (2013) }\end{array}$ & & $\mathrm{X}$ & \\
\hline $\begin{array}{l}\text { Pants on fire: The electrophysiological signature of telling } \\
\text { a lie. Pfister et al. (2014) }\end{array}$ & & & $\mathrm{X}$ \\
\hline $\begin{array}{l}\text { Detection of concealed information using event-related } \\
\text { potentials. Terola et al. (2014) }\end{array}$ & & & $X$ \\
\hline $\begin{array}{l}\text { A review of the collective interviewing approach to } \\
\text { detecting deception in pairs. } \\
\text { Vernham \& Vrij. (2015) }\end{array}$ & $\mathrm{X}$ & & \\
\hline $\begin{array}{l}\text { Measuring the cognitive resources consumed per second } \\
\text { for real-time lie-production and recollection: a dual- } \\
\text { tasking paradigm. } \\
\text { Hu et al. (2015) }\end{array}$ & $\mathrm{X}$ & & \\
\hline
\end{tabular}




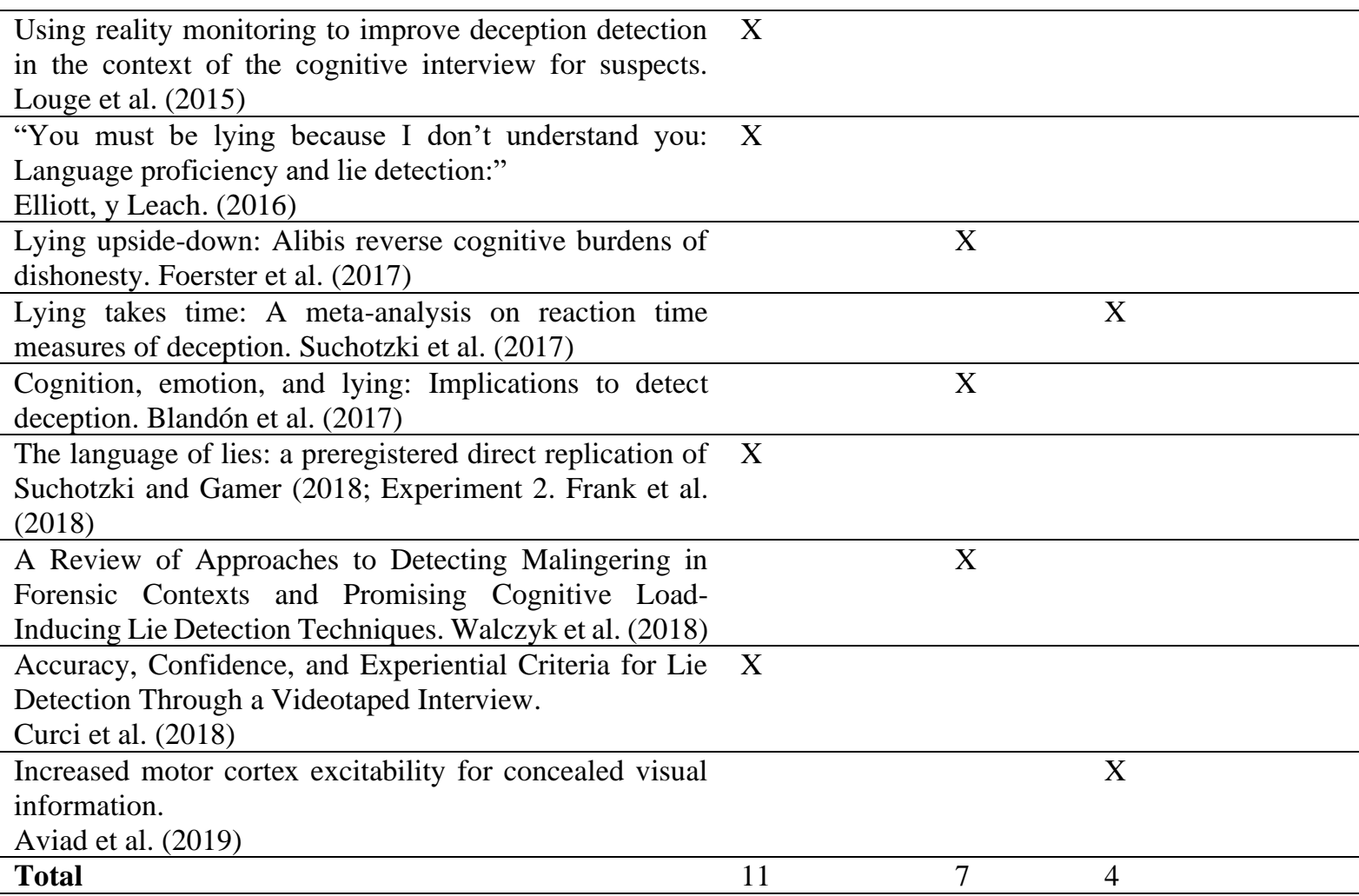

Dentro de esta primera tabla que sintetiza los resultados de los 22 artículos de investigación obtenidos, se puede apreciar a 11 artículos que confirman la idea que la carga cognitiva pueda servir de indicador al momento de intentar detectar las mentiras dentro de discurso, por otro lado, se obtuvo 7 artículos niegan la afirmación anterior, mientras 4 mencionan que los resultados que obtuvieron no fueron concluyentes.

\subsection{CARGA COGNITIVA Y DILATACIÓN PUPILAR.}

Como se mencionó con anterioridad; la literatura científica liga a la carga cognitiva con la mentira, al revisar la información obtenida para profundizar en el tema mencionado se encontró evidencia que permite pensar que existe una relación directa entre la dilatación pupilar y la carga cognitiva. Iqbal., et al. (2004), señala que de acuerdo al nivel de la carga cognitiva se puede ver el reflejo involuntario de aumento o disminución del tamaño pupilar, dicho en otras palabras, se podría detectar de una manera eficaz la mentira en las personas gracias a la oculometría.

Berrien y Huntington en 1943, dan a conocer los resultados obtenidos a través de su investigación sobre la efectividad para detectar el engaño mediante la técnica que centra en el estudio en la dilatación de pupilas a personas que fueron culpadas de crímenes, se obtiene una puntuación de $48 \%$ de efectividad para identificar a personas que emiten respuestas falsas, en cambio un $18 \%$ de efectividad para detectar a personas que decían la verdad. 
En los años siguientes Lubow y Fein (1996), en un experimento realizado afirman que el método de detección de la mentira por la dilatación pupilar, alcanza un grado de precisión de un $70 \%$ en las personas con conocimientos de culpabilidad, mientras tanto, en las personas con información verdadera se logra detectar en un $100 \%$ en base a estos resultados se observa un gran índice de fiabilidad al aplicar estas técnicas en la detección del engaño.

Dentro del ámbito de la salud se observa caso de personas que llegan a consulta refiriendo padecer algún tipo de patología con la finalidad de obtener algún beneficio económico por lo que Heaver y Hutton (2010). Desarrollaron su investigación para detectar la mentira de estos falsos pacientes con el principio de que una persona que miente sufre una dilatación pupilar, los resultados de esta investigación fueron alentadores por lo que sugiere que este principio tiene gran potencial dentro del ámbito clínico para detectar si el paciente está mintiendo.

Hacker et al. (2014). En su propuesta para detectar el engaño basado en el diámetro pupilar el autor señala que logra alcanzar los siguientes porcentajes, más del $85 \%$ de los casos se clasificaron correctamente en los estudios de laboratorio y el 77,7\% de los casos se clasificaron correctamente en uno de los estudios de campo, por lo que concluye que el uso de la respuesta pupilar y los movimientos oculares durante la lectura promete como una nueva herramienta para la detección del engaño.

Jeffrey eta al. (2016), menciona que la oculometria como indicador del engaño evoluciona de distintas maneras ante un estímulo, y estos cambios sirven para identificar el engaño dentro de un testimonio de manera independiente de cualquier estimulo.

Proudfoot et al. (2016), en su investigación para determinar la evolución en la medida pupilar en el sistema de detección de la mentira, los resultados obtenidos indican que la medida ocular de las personas que engañan cambia de una manera diferente y que estos cambios podrían servir como indicadores del engaño siempre y cuando se muestren estímulos pertinentes.

Continuando con las investigaciones que ha llevado a cabo en búsqueda de la relación entre dilatación pupilar y mentira, Choi et al. (2016), logra identificar en alumnos del grupo de culpables un aumento de la pupila de un 40,2\% en relación al grupo de estudiantes inocentes, esto le hace llegar a la conclusión que sería capaz de detectar a los mentirosos utilizando la dilatación pupilar y agregando la respuesta de la conducta de la piel

Investigaciones anteriores indican que la dilatación de la pupila es un indicador de la mentira por lo que, Nurçina et al. (2017), realiza un experimento donde capturan la dilatación de las pupilas ante discursos de verdad y mentira, con lo que están entrenando a una red neural para que logre identificar las mentiras de forma automática. 
Con la finalidad de lograr identificar las variables que podrían servir como indicadores de engaño Cho y lee (2018), han puesto a prueba la hipótesis que indica que la dilatación pupilar es mayor ante un aumento de la carga cognitiva siendo como resultado de una mentira, sin embargo, los resultados sugieren que en efecto hay una dilatación pupilar ante el aumento de la carga cognitiva, pero dicho no es único del engaño sino que también se presenta ante una excitación emocional, por esta razón los autores recomienda seguir investigando para llegar a un procedimiento que permita usar esta variable como indicador fiable del engaño.

Uno de los instrumentos más utilizados en todo el mundo para determinar si una persona dice la verdad es el polígrafo, ha sido criticado por muchos autores quienes comentan que se puede modificar los resultados mediante un control de la respiración y la tensión en general, por lo que Lee y Lee (2018), en su búsqueda de encontrar cual es la mejor herramienta para determinar la veracidad del discurso, se centra en la medición del diámetro de la pupila poniendo a prueba en simultaneo al polígrafo y a la dilatación de la pupila, esto le llevo a la conclusión que la dilatación en las personas culpables fueron detectables por lo que sugiere que el uso de la oculometría servirá como un complemento para el polígrafo.

Para determinar una mentira es necesario estudiar múltiples variables que puedan contribuir a identificar el engaño, por lo que Ge et al. (2020), se centró en el estudio de la dilatación de la pupila, la duración media de la fijación para determinar si una persona está mintiendo, después de su estudio con 40 personas llega a la conclusión que estas dos variables sirven para identificar el engaño dentro de un discurso.

Tabla 2 .

CARGA COGNITIVA Y DILATACIÓN PUPILAR.

\begin{tabular}{lll}
\hline NOMBRE DEL ARTICULO. & $\begin{array}{l}\text { CONFIRM } \\
\text { A. }\end{array}$ & $\begin{array}{l}\text { NIEGA. } \\
\text { NO } \\
\text { CONCLUYEN }\end{array}$ \\
\hline $\begin{array}{l}\text { Task evoked pupil response to mental workload in human- } \\
\text { computer interaction. Iqbal et al. (2004) }\end{array}$ & $\mathrm{X}$ & \\
\hline $\begin{array}{l}\text { An exploratory study of pupillary responses during deception. } \\
\text { Berrien y Huntington (1943) }\end{array}$ & $\mathrm{X}$ & \\
\hline $\begin{array}{l}\text { Pupillary size in response to a visual guilty knowledge test: } \\
\text { New technique for the detection of deception. Lubow y Fein } \\
\text { (1996) }\end{array}$ & $\mathrm{X}$ \\
\hline $\begin{array}{l}\text { Keeping an eye on the truth: Pupil size, recognition memory } \\
\text { and malingering. Heaver y Hutton. 2010 }\end{array}$ & $\mathrm{X}$ \\
\hline $\begin{array}{l}\text { Detecting Deception Using Ocular Metrics During Reading. } \\
\text { Hacker et al. (2014) }\end{array}$ & $\mathrm{X}$ \\
\hline $\begin{array}{l}\text { More Than Meets the Eye: How Oculometric Behaviors Evolve } \\
\text { Over the Course of Automated Deception Detection }\end{array}$ & $\mathrm{X}$ \\
Interactions. Jeffrey et al. (2016) & & \\
\hline $\begin{array}{l}\text { A Possibility of Pupil Diameter as Reliable Index for Lie } \\
\text { Detection. Choi et al. (2016) }\end{array}$ & $\mathrm{X}$ & \\
\hline $\begin{array}{l}\text { Lie detection on pupil size by back propagation neural network. } \\
\text { Nurçina et al. (2017) }\end{array}$ & $\mathrm{X}$ & \\
\hline
\end{tabular}


A Comparison of Cognitive Load and Emotional Arousal on $\mathrm{X}$ Liar's Pupil Diameter Changes. Cho y lee (2018)

Increase of Lie Detection Accuracy through Integrated $\mathrm{X}$

Measurement of Pupil Dilation and Polygraph. Lee y Lee (2018)

Application of Eye Tracker in Lie Detection. $\quad \mathrm{X}$

Ge et al. (2020)

Total $\quad 10 \quad 1001$

En la segunda tabla que interpreta los resultados de los artículos recolectados para identificar si la dilatación de la pupila podría funcionar como indicador del engaño se obtuvo un total de 11 artículos de investigación que lograron cumplir con los criterios de inclusión, se obtuvieron 10 artículos que confirman el postulado antes mencionado, por lo contrario, solo 1 artículo manifiesta que los resultados no fueron concluyentes.

\section{MOVIMIENTO OJOS Y LA MENTIRA.}

Movimientos sacádicos, son entendidos por Louis Emile Javal (1839-1907) en Rincón., et al, (2016, p.258) como "los movimientos de desplazamientos oculares rápidos horizontales y transversales, a manera de saltos, efectuados durante la lectura."

En las investigaciones relacionadas a las mentiras podemos encontrar a la realizada por Cardona \& Quevedo (2003), en la cual maneja la hipótesis que a mayor carga cognitiva se evidencia mayor número de movimientos oculares sacádicos y luego la relacionamos con la teoría de que las personas que mienten muestran mayor número de sacadas, el experimento nos demostró lo contrario ya que no se observó diferencias significativas durante el aumento de complejidad para decir que en verdad las sacadas puedan funcionar como indicadores de la veracidad en el discurso.

Con la teoría de que las personas que mienten experimentan una mayor carga cognitiva cuando se realiza un control de los elementos claves del discurso, siendo lo contrario de los elementos de control, con esta primicia se postula la idea que las personas que están respondiendo ante elementos claves del discurso hay una disminución del número de parpadeos, lo que Leal y Vrij, 2010. Concluyen que al momento de realizar análisis del discurso a una persona que no miente, debería observarse el mismo número de parpadeos ante la respuesta de elementos claves y de control.

Las investigaciones realizadas por Fisher (2010), han puesto a los movimientos de los ojos como indicadores fiables del engaño, ya que en sus experimentos en un $88 \%$ las personas pueden identificar a un mentiroso gracias a los movimientos de los ojos, por esta razón el autor manifiesta que es posible identificar una mentira dentro de un discurso, además reconoce la necesidad de trabajar con otras variables como la dilatación pupilar y la carga cognitiva. 
En dos experimentos realizados en 2012 por Cook, et al. Indicaron la existencia de dos procesos cognitivos implicados en el engaño, estos son la vigilancia y la estrategia, los cuales pueden ser reflejados en diferentes medidas por el movimiento motor ocular, por lo tanto, afirma que se puede detectar el engaño si se cuenta con una buena técnica para la detección de movimientos sacádicos.

Wiseman et al. (2012), en su intento de comprobar la teoría de la PNL, de que las personas mueven los ojos a diferentes lugares cuando dicen mentiras, decidió crean tres experimentos en los cuales no se descubrieron diferencias significativas, por lo que los resultados de los estudios no concuerdan con la teoría de la PNL de los movimientos oculares.

Los estudios realizados por Taniguchi y Ono (2013), donde realizan un rastreo de los movimientos de los ojos sin contacto, los resultados sugieren que podrían ser utilizados un grado de eficacia como indicadores del engaño.

Durante una investigación con 40 personas para determinar si los movimientos de los ojos servían como indicadores del engaño, realizo un rastreo de los movimientos de los ojos después de un delito simulado, donde los resultados no mostraron diferencias estadísticas significativas, por lo tanto, Ge et al. (2020), no pudo confirmar que los movimientos de los ojos puedan funcionar como un indicador claro del engaño.

\begin{tabular}{|c|c|c|}
\hline NOMBRE DEL ARTICULO. & $\begin{array}{l}\text { CONFIRM } \\
\text { A. }\end{array}$ & NIEGA. NO CONCLUYENTE \\
\hline $\begin{array}{l}\text { Blinking and Driving: the Influence of Saccades and } \\
\text { Cognitive Workload. Cardona \& Quevedo (2013) }\end{array}$ & & $\mathrm{X}$ \\
\hline $\begin{array}{l}\text { The occurrence of eye blinks during a guilty } \\
\text { knowledge test. Leal y Vrij (2010) }\end{array}$ & $\mathrm{X}$ & \\
\hline $\begin{array}{l}\text { If an avatar lies, you can tell by its eyes. } \\
\text { Fisher (2010) }\end{array}$ & $\mathrm{X}$ & \\
\hline $\begin{array}{l}\text { Lyin' eyes: Ocular-motor measures of reading reveal } \\
\text { deception. Cook et al. (2012) }\end{array}$ & $X$ & \\
\hline $\begin{array}{l}\text { The eyes don't have it: lie detection and Neuro- } \\
\text { Linguistic Programming. Wiseman et al. (2012) }\end{array}$ & & $\mathrm{X}$ \\
\hline $\begin{array}{l}\text { Non-contact measurement of eye movements in the } \\
\text { detection of deception. } \\
\text { Taniguchi y Ono (2013) }\end{array}$ & $\mathrm{X}$ & \\
\hline $\begin{array}{l}\text { Application of Eye Tracker in Lie Detection. } \\
\text { Ge et al. (2020) }\end{array}$ & & $\mathrm{X}$ \\
\hline Total & 4 & 0 \\
\hline
\end{tabular}

En cuanto a las últimas variables, se lograron reunir 7 artículos que hablan del tema de los cuales 4 confirman y los 3 artículos restantes niegan la posibilidad que los movimientos sacádicos puedan servir como indicadores del engaño, en este apartado no se identifica investigaciones que no sean concluyentes. 


\section{CONCLUSIÓN:}

En la actualidad la ciencia intenta buscar una manera efectiva de identificar si una persona miente o dice la verdad para lo que se ha invertido mucho dinero en investigaciones de grandes empresas, por lo tanto, en este la investigación profunda de artículos de investigación pudimos determinando cuan efectivos son las variables que usan las empresas para desarrollar estas nuevas tecnologías.

En cuanto a la variable de carga cognitiva, se observa resultados similares por lo que se concluye que no sería útil para determinar la mentira dentro de un discurso, los porcentajes que se obtiene son de $45 \%$ que afirman, el $35 \%$ niegan y el $20 \%$ no son concluyentes, por esta razón se descarta la idea de que se pueda usar la carga cognitiva como un indicador de veracidad.

Dentro del apartado de la dilatación pupilar es factible decir que existe evidencia que se puede utilizar como un indicador fiable, ya que las investigaciones que lo afirman alcanzan un 92,3\% y tan solo el 7,7\%, sin embargo hay que tener en cuenta que la muestra de artículos es baja, además podemos encontrar en la investigación de Martes et al. (2016), sobre la dilatación de la pupila a los estímulos sexuales explícitos y no explícitos demuestran que la pupila se dilata de la misma manera que cuando una persona miente, por lo tanto, es importante seguir estudiante este apartado controlando todas las variables para evitar errores.

Si hablamos de los movimientos sacádicos, hay que tener en cuenta su poco material bibliográfico para realizar este análisis, además se puede decir que los resultados están divididos en porcentajes parecidos entre el $57,1 \%$ y el $42,9 \%$, por lo tanto, no se puede afirmar que los movimientos de los ojos puedan servir de indicadores del engaño.

Por último, al contar con la información necesaria obtenida de los artículos a través de las revistas seleccionados podemos llegar a la conclusión que la variable movimientos sacádicos y carga cognitiva no muestran evidencia suficiente para considerar que pueden funcionar como indicadores en la veracidad del testimonio, sin embargo la variable dilatación pupilar muestra mejores evidencias para cumplir con el objetivo de indicador de la verdad, pero es necesario investigar más e implementar más tecnología para una correcta medición además de ponerlo a prueba en un ambiente que sea completamente controlado para evitar la interferencias de factores como la luz, alteraciones emocionales e incluso estímulos sexuales que pudieran dar un falso positivo al momento de usar la dilatación de la pupila para detectar una mentira. 


\section{REFERENCIAS BIBLIOGRÁFICAS}

- Watts, M. M., Holmes, L., Savin R. C., Rieger. G. (2016). Pupil dilation to explicit and nonexplicit sexual stimuli. SPRINGER LINK, volume (46), 155 - 165. https://doi.org/10.1007/s10508-0160801-8

- Hadar, A. A., Lazarovits, A., \& Yarrow, K. (2019). Increased motor cortex excitability for concealed visual information. Journal of Psychophysiology, 33(4), 286295. https://doi.org/10.1027/0269-8803/a000230

- $\quad$ Foerster, A., Wirth, R., Herbort, O., Kunde, W., \& Pfister, R. (2017). Lying upside-down: Alibis reverse cognitive burdens of dishonesty. Journal of Experimental Psychology: Applied, 23(3), 301319. https://doi.org/10.1037/xap0000129

- $\quad$ Suchotzki, K., Verschuere, B., Van Bockstaele, B., Ben-Shakhar, G., \& Crombez, G. (2017). Lying takes time: A meta-analysis on reaction time measures of deception. Psychological Bulletin, 143(4), 428-453. https://doi.org/10.1037/bu10000087

- $\quad$ Elliott, Elizabeth, Leach, Amy-May. 2016. "You must be lying because I don't understand you: Language proficiency and lie detection:" Correction to Elliott and Leach (2016) (2017). Journal of Experimental Psychology: Applied, 23(1), 99. https://doi.org/10.1037/xap0000121

- $\quad$ Vrij, A., Mann, S. A., Fisher, R. P., Leal, S., Milne, R., \& Bull, R. (2008). Increasing cognitive load to facilitate lie detection: The benefit of recalling an event in reverse order. Law and Human Behavior, 32(3), 253-265. https://doi.org/10.1007/s10979-007-9103-y

- $\quad$ Cook, A. E., Hacker, D. J., Webb, A. K., Osher, D., Kristjansson, S. D., Woltz, D. J., \& Kircher, J. C. (2012). Lyin' eyes: Ocular-motor measures of reading reveal deception. Journal of Experimental Psychology: Applied, 18(3), 301-313. https://doi.org/10.1037/a0028307

- $\quad$ Lubow, R. E., \& Fein, O. (1996). Pupillary size in response to a visual guilty knowledge test: New technique for the detection of deception. Journal of Experimental Psychology: Applied, 2(2), 164177. https://doi.org/10.1037/1076-898X.2.2.164

- $\quad$ Berrien, F. K., \& Huntington, G. H. (1943). An exploratory study of pupillary responses during deception. Journal of Experimental Psychology, 32(5), 443-449. https://doi.org/10.1037/h0063488

- $\quad$ Logue, M., Book, A. S., Frosina, P., Huizinga, T., \& Amos, S. (2015). Using reality monitoring to improve deception detection in the context of the cognitive interview for suspects. Law and Human Behavior, 39(4), 360-367. https://doi.org/10.1037/lhb0000127

- Thomas, H. F., \&Melissa, J. Y. (2009). Self-reported cues about deceptive and truthful communication: The effects of cognitive capacity and communicator veracity. www.tandfonline.com, 48(2), 101-119. https://doi.org/10.1080/01463370009385585 
- $\quad$ Bembibre, J. \& Higueras, L. (2009). Differential effectiveness of the cognitive interview in a simulation of testimony. $\quad$ www.tandfonline.com, $17(6), \quad 473-489$. https://doi.org/10.1080/10683160903321540

- $\quad$ Mann, S. \& Vrij, A. (2007). Police officers' judgements of veracity, tenseness, cognitive load and attempted behavioural control in real-life police interviews . www.tandfonline.com, 12(3),307- 319. https://doi.org/10.1080/10683160600558444

- $\quad$ Pfister, R. Foerster, A. \& Kunde, W. (2014). Pants on fire: The electrophysiological signature of telling a lie. www.tandfonline.com, 9(6), 562-572. https://doi.org/10.1080/17470919.2014.934392

- Leal, S. \& Vrij, A. (2010). The occurrence of eye blinks during a guilty knowledge test. www.tandfonline.com, 9(6), 562-572. https://doi.org/10.1080/10683160902776843

- $\quad$ Frank, A. Biberci, S. \& Verschuere, B. (2018). The language of lies: a preregistered direct replication of Suchotzki and Gamer (2018; Experiment 2). www.tandfonline.com, 33(6), 1310- 1315. https://doi.org/10.1080/02699931.2018.1553148

- $\quad$ Bembibre, J. \& Higueras. L. (2011). Comparative analysis of true or false statements with the source monitoring model and the cognitive interview: special features of the false accusation of innocent people. www.tandfonline.com, 18(10), 913-928. https://doi.org/10.1080/1068316X.2011.589387

- $\quad$ Vernham, Z., \& Vrij, A. (2015). A review of the collective interviewing approach to detecting deception in pairs. www.tandfonline.com, 1(1), 43- 58. https://doi.org/10.1080/23744006.2015.1051756

- Dukala, K., Ludwig, S., \& Romuald Polczyk. (2008). Detecting deception: does the cognitive interview impair discrimination with CBCA criteria in elderly witnesses? www.tandfonline.com, 8(1), 89111. https://doi.org/10.1300/J070v08n01_06

- Cardona, G., \& Quevedo, N. (2013). Blinking and Driving: the Influence of Saccades and Cognitive Workload. www.tandfonline.com, 244. https://doi.org/10.3109/02713683.2013.841256

- $\quad$ Blandón-Gitlin, I., López, R. M., Masip, J., \& Fenn, E. (2017). Cognition, emotion, and lying: Implications to detect deception. Anuario de Psicología Jurídica, 27(1), 95-106. https://doi.org/10.1016/j.apj.2017.02.004

- Terol, O., Álvarez, M., Melgar, N., \& Manzanero, A. L. (2014). Detection of concealed information using event-related potentials. Anuario de Psicología Jurídica, 24(1), 4955. https://doi.org/10.1016/j.apj.2014.06.004.

- Wiseman, R., Watt, C., ten Brinke, L., Porter, S., Couper, S.-L., \& Rankin, C. (2012). The Eyes Don't Have It: Lie Detection and Neuro-Linguistic Programming. PLoS ONE, 7(7), e40259. https://doi.org/10.1371/journal.pone.0040259

- $\quad$ Walczyk, J. J., Igou, F. P., Dixon, A. P., \& Tcholakian, T. (2013). Advancing Lie Detection by Inducing Cognitive Load on Liars: A Review of Relevant Theories and Techniques Guided by Lessons 
from Polygraph-Based Approaches. Frontiers in Psychology, 4. https://doi.org/10.3389/fpsyg.2013.00014

- $\quad \mathrm{Hu}, \mathrm{C} .$, Huang, K., Hu, X., Liu, Y., Yuan, F., Wang, Q., \& Fu, G. (2015). Measuring the cognitive resources consumed per second for real-time lie-production and recollection: a dual-tasking paradigm. Frontiers in Psychology, 6, 1-7. https://doi.org/10.3389/fpsyg.2015.00596

- $\quad$ Curci, A., Lanciano, T., Battista, F., Guaragno, S., \& Ribatti, R. M. (2019). Accuracy, Confidence, and Experiential Criteria for Lie Detection Through a Videotaped Interview. Frontiers in Psychiatry, 9. https://doi.org/10.3389/fpsyt.2018.00748

- Van Bockstaele, B., Verschuere, B., Moens, T., Suchotzki, K., Debey, E., \& Spruyt, A. (2012). Learning to Lie: Effects of Practice on the Cognitive Cost of Lying. Frontiers in Psychology, 3. https://doi.org/10.3389/fpsyg.2012.00526

- Walczyk, J. J., Sewell, N., \& DiBenedetto, M. B. (2018). A Review of Approaches to Detecting Malingering in Forensic Contexts and Promising Cognitive Load-Inducing Lie Detection Techniques. Frontiers in psychiatry, 9, 700. https://doi.org/10.3389/fpsyt.2018.00700

- $\quad$ Proudfoot, J. G., Jenkins, J. L., Burgoon, J. K., \& Nunamaker, J. F. (2016). More Than Meets the Eye: How Oculometric Behaviors Evolve Over the Course of Automated Deception Detection Interactions. Journal of Management Information Systems, 33(2), 332360. https://doi.org/10.1080/07421222.2016.1205929.

- $\quad$ Nurçin, F. V., Imanov, E., Işın, A., \& Ozsahin, D. U. (2017). Lie detection on pupil size by back propagation neural network. Procedia Computer Science, 120, 417421. https://doi.org/10.1016/j.procs.2017.11.258

- $\quad$ Hacker, D. J., Kuhlman, B. B., Kircher, J. C., Cook, A. E., \& Woltz, D. J. (2014). Detecting Deception Using Ocular Metrics During Reading. Credibility Assessment, 159216. https://doi.org/10.1016/b978-0-12-394433-7.00005-1

- $\quad$ Cho, B. A. A., \& Lee, J.-H. (2018). A Comparison of Cognitive Load and Emotional Arousal on Liar's Pupil Diameter Changes. International Journal of Psychophysiology, 131, S74. https://doi.org/10.1016/j.ijpsycho.2018.07.214

- $\quad$ Choi, H., Kim, S., Lee, S.-H., \& Lee, J.-H. (2016). A Possibility of Pupil Diameter as Reliable Index for Lie Detection. International Journal of Psychophysiology, 108, 150. https://doi.org/10.1016/j.ijpsycho.2016.07.435

- $\quad$ Heaver, B., \& Hutton, S. B. (2010). Keeping an eye on the truth: Pupil size, recognition memory and malingering. International Journal of Psychophysiology, 77(3), 306-306. https://doi.org/ 10.1016/j.ijpsycho.2010.06.206. 
- Lee, E. J., \& Lee, J. H. (2018). Increase of Lie Detection Accuracy through Integrated Measurement of Pupil Dilation and Polygraph. International Journal of Psychophysiology, 131, S124S125. https://doi.org/ 10.1016/j.ijpsycho.2018.07.336

- Fisher, R. (2010). Avatars' lies betrayed by their eyes. New Scientist, 206(2755), 20. https://doi.org/ 10.1016/s0262-4079(10)60859-7

- $\quad$ Ge, F. F., Yang, X. Q., Chen, Y. X., Huang, H. L., Shen, X. C., Li, Y., \& Hu, J. M. (2020). Application of Eye Tracker in Lie Detection. Fa yi xue za zhi,36(2), 229-232. https://doi.org/10.12116/j.issn.1004-5619.2020.02.015.

- $\quad$ Taniguchi, Y., \& Ono, Y. (2013). Non-contact measurement of eye movements in the detection of deception. The Japanese Journal of Psychology, 84(1), 10-19. https://doi.org/10.4992/jjpsy.84.10

- $\quad$ Iqbal, S. T., Zheng, X, S., Y Bailey, B, P. (2004). Task evoked pupil response to mental workload in human-computer interaction. Proceedings of the Conference on Human Factors in Computing Systems, 2004(1), 1477- 1480. https://doi.org/10.1145 / 3314111.3319831

- $\quad$ Snowden, RJ, McKinnon, A., Fitoussi, J. y Gray, NS (2017). Respuestas pupilares a imágenes estáticas de hombres y mujeres: ¿una posible medida del interés sexual? www.tandfonline.com, 111. https://doi.org/10.1080 / 00224499.2017.1394959

- $\quad$ Snowden, R. J., McKinnon, A., Fitoussi, J., \& Gray, N. S. (2017). Pupillary Responses to Static Images of Men and Women: A Possible Measure of Sexual Interest? The Journal of Sex Research, 111. doi:10.1080/00224499.2017.1394959 
Anexo:

\begin{tabular}{|c|c|c|c|c|c|c|c|c|c|}
\hline No. & $\begin{array}{l}\text { BASE } \\
\text { DE } \\
\text { DATOS }\end{array}$ & AUTOR & AÑO & DOI & TÍTULO & OBJETIVO & MÉTODO & RESULTADO & $\begin{array}{l}\text { ASPECTO } \\
\text { DISCUTIDO }\end{array}$ \\
\hline 1 & $\begin{array}{l}\text { psycnet.a } \\
\text { pa.org }\end{array}$ & $\begin{array}{l}\text { Aviad et } \\
\text { al. }\end{array}$ & 2019 & $\begin{array}{l}\frac{\frac{\text { https:// }}{\text { doi.org/ }}}{10.102} \\
\frac{7 / 0269-}{7803 / \mathrm{a}} \\
\underline{000230}\end{array}$ & $\begin{array}{l}\text { Increased } \\
\text { motor cortex } \\
\text { excitability } \\
\frac{\text { for }}{\text { concealed }} \\
\frac{\text { visual }}{\text { information. }}\end{array}$ & $\begin{array}{l}\text { Examinar si la } \\
\text { excitabilidad puede } \\
\text { ser usada para sugerir } \\
\text { la presencia de } \\
\text { información } \\
\text { deliberadamente } \\
\text { oculta en una versión } \\
\text { modificada del test de } \\
\text { conocimiento de } \\
\text { culpabilidad (GKT) }\end{array}$ & $\begin{array}{l}\text { Los participantes presionaron las } \\
\text { teclas para indicar, ya sea con } \\
\text { veracidad o engaño, su } \\
\text { familiaridad con una serie de } \\
\text { rostros. Se registraron los } \\
\text { potenciales evocados por el motor } \\
\text { (MEP) durante la preparación de } \\
\text { la respuesta para medir la } \\
\text { excitabilidad neural específica del } \\
\text { músculo. }\end{array}$ & $\begin{array}{l}\text { Informamos de un } \\
\text { aumento a nivel de grupo } \\
\text { de la excitabilidad } \\
\text { corticoespinal general de } \\
300 \text { ms tras el inicio del } \\
\text { estímulo durante la } \\
\text { condición engañosa, sin } \\
\text { activación específica de } \\
\text { la representación neural } \\
\text { del dedo que dice la } \\
\text { verdad. }\end{array}$ & $\begin{array}{l}\text { Discutimos los procesos } \\
\text { cognitivos, } \\
\text { particularmente } \\
\text { conflicto de respuesta y/o } \\
\text { las respuestas } \\
\text { automatizadas a } \\
\text { estímulos familiares, que } \\
\text { pueden impulsar el } \\
\text { aumento inespecífico } \\
\text { observado de la } \\
\text { excitabilidad motora en } \\
\text { el engaño. }\end{array}$ \\
\hline 2 & $\begin{array}{l}\text { psycnet.a } \\
\text { pa.org }\end{array}$ & $\begin{array}{l}\text { Foerster } \\
\text { et al. }\end{array}$ & 2017 & $\begin{array}{l}\frac{\underline{\text { https:// }}}{\underline{\text { doi.org/ }}} \\
\frac{10.103}{7 / \mathrm{xap0}} \\
\underline{\text { 000129 }}\end{array}$ & $\begin{array}{l}\frac{\text { Lying }}{\text { upside- }} \\
\frac{\text { down: Alibis }}{\text { reverse }} \\
\frac{\text { cognitive }}{\text { burdens of }} \\
\text { dishonesty. }\end{array}$ & $\begin{array}{l}\text { Sugerir que las falsas } \\
\text { coartadas explícitas } \\
\text { podrían tener un } \\
\text { impacto considerable } \\
\text { en estas operaciones } \\
\text { cognitivas. }\end{array}$ & $\begin{array}{l}\text { Los participantes primero } \\
\text { realizaron varias tareas en una } \\
\text { misión preexperimental y } \\
\text { recibieron una falsa coartada } \\
\text { después. La falsa coartada } \\
\text { establecía acciones alternativas } \\
\text { que los participantes tenían que } \\
\text { fingir haber realizado en lugar de } \\
\text { las acciones realmente realizadas. }\end{array}$ & $\begin{array}{l}\text { Las falsas coartadas } \\
\text { pueden anular por } \\
\text { completo las actividades } \\
\text { realmente realizadas y, } \\
\text { por lo tanto, documenta } \\
\text { una grave limitación para } \\
\text { los enfoques cognitivos } \\
\text { de la detección de } \\
\text { mentiras. }\end{array}$ & $\begin{array}{l}\text { Los procesos cognitivos } \\
\text { que subyacen a la } \\
\text { deshonestidad, } \\
\text { especialmente la } \\
\text { inhibición de las } \\
\text { tendencias de respuesta } \\
\text { honesta automática, se } \\
\text { reflejan en los tiempos de } \\
\text { respuesta y otras medidas } \\
\text { de comportamiento. }\end{array}$ \\
\hline 3 & $\begin{array}{l}\text { psycnet.a } \\
\text { pa.org }\end{array}$ & $\begin{array}{l}\text { Suchotzk } \\
\text { i et al. }\end{array}$ & 2017 & $\begin{array}{l}\frac{\text { https:// }}{\text { doi.org/ }} \\
\underline{10.103} \\
\underline{7 / \mathrm{bul00}} \\
\underline{00087}\end{array}$ & 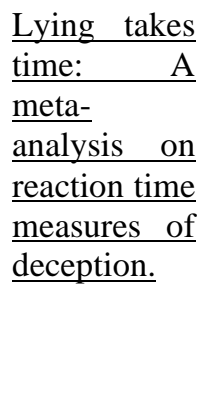 & $\begin{array}{l}\text { Desentrañar los } \\
\text { mecanismos } \\
\text { cognitivos que } \\
\text { subyacen al engaño y } \\
\text { por desarrollar una } \\
\text { teoría integral del } \\
\text { engaño. }\end{array}$ & $\begin{array}{l}\text { En este documento se presentan } \\
\text { los resultados de un metaanálisis } \\
\text { de } 114 \text { estudios }(\mathrm{n}=3307) \text { que } \\
\text { utilizan paradigmas de RT } \\
\text { computarizados para evaluar el } \\
\text { costo cognitivo de la mentira. }\end{array}$ & $\begin{array}{l}\text { Los resultados } \\
\text { corroboraron los actuales } \\
\text { enfoques cognitivos del } \\
\text { engaño, la } \\
\text { heterogeneidad } \\
\text { observada exige una } \\
\text { mayor investigación } \\
\text { sobre las condiciones } \\
\text { límite del coste cognitivo } \\
\text { del engaño. }\end{array}$ & $\begin{array}{l}\text { Esta situación ha dado } \\
\text { lugar a un aumento de los } \\
\text { esfuerzos por desentrañar } \\
\text { los mecanismos } \\
\text { cognitivos que subyacen } \\
\text { al engaño y por } \\
\text { desarrollar una teoría } \\
\text { integral del engaño. }\end{array}$ \\
\hline 4 & $\begin{array}{l}\text { psycnet.a } \\
\text { pa.org }\end{array}$ & $\begin{array}{l}\text { Elliott, y } \\
\text { Leach. }\end{array}$ & 2016 & $\frac{\text { https:// }}{\text { doi.org/ }}$ & $\begin{array}{ll}\text { "You } & \text { must } \\
\text { be } & \text { lying }\end{array}$ & $\begin{array}{l}\text { Informar de un error } \\
\text { en "Debes estar }\end{array}$ & $\begin{array}{l}\text { Los observadores }(\mathrm{N}=132) \\
\text { fueron asignados al azar para }\end{array}$ & $\begin{array}{l}\text { Estos resultados sugieren } \\
\text { que entrevistar a las }\end{array}$ & $\begin{array}{l}\text { "Teoría de la detección } \\
\text { de señales" }\end{array}$ \\
\hline
\end{tabular}




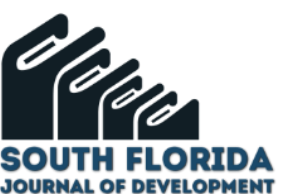

\begin{tabular}{|c|c|c|c|c|c|c|c|c|c|}
\hline & & & & $\frac{\frac{10.103}{7 / \mathrm{xap} 0}}{\underline{000121}}$ & $\begin{array}{l}\text { because I } \\
\text { don't } \\
\text { understand } \\
\text { you: } \\
\text { Language } \\
\text { proficiency } \\
\text { and lie } \\
\text { detection:" } \\
\text { Correction to } \\
\text { Elliott and } \\
\text { Leach } \\
\text { (2016). }\end{array}$ & $\begin{array}{lr}\text { mintiendo porque no } \\
\text { te entiendo: Dominio } \\
\text { del idioma y } \\
\text { detección } & \text { de } \\
\text { mentiras" } & \text { de } \\
\text { Elizabeth Elliott } & \text { y } \\
\text { Amy-May. } & \end{array}$ & $\begin{array}{l}\text { hacer juicios de engaño sobre los } \\
\text { entrevistados }(\mathrm{N}=56) \text { de cuatro } \\
\text { grupos de competencia (es decir, } \\
\text { nativos, avanzados, intermedios y } \\
\text { principiantes de habla inglesa). }\end{array}$ & $\begin{array}{l}\text { personas en sus idiomas } \\
\text { no nativos puede crear } \\
\text { desigualdades en el } \\
\text { sistema de justicia. }\end{array}$ & \\
\hline 5 & $\begin{array}{l}\text { psycnet.a } \\
\text { pa.org }\end{array}$ & Vrij et al. & 2008 & $\frac{\frac{\text { https:// }}{\text { doi.org/ }}}{\frac{10.100}{\frac{7 / \mathrm{s} 1097}{9-007-}}}$ & $\begin{array}{l}\text { Increasing } \\
\begin{array}{l}\text { cognitive } \\
\text { load to }\end{array} \\
\text { facilitate lie } \\
\text { detection: } \\
\text { The benefit } \\
\text { of recalling } \\
\text { an event in } \\
\text { reverse } \\
\text { order. }\end{array}$ & $\begin{array}{l}\text { Probar la hipótesis de } \\
\text { la detección de } \\
\text { mentira a través del } \\
\text { relato inverso de las } \\
\text { historias. }\end{array}$ & $\begin{array}{l}\text { Pedir a los sospechosos que } \\
\text { transmitieran sus historias en } \\
\text { orden inverso mejoró la } \\
\text { capacidad de los observadores } \\
\text { policiales para detectar el engaño } \\
\text { y no dio lugar a un sesgo de } \\
\text { respuesta. }\end{array}$ & $\begin{array}{l}\text { Pedir a los sospechosos } \\
\text { que transmitieran sus } \\
\text { historias en orden inverso } \\
\text { mejoró la capacidad de } \\
\text { los observadores } \\
\text { policiales para detectar el } \\
\text { engaño y no dio lugar a } \\
\text { un sesgo de respuesta. }\end{array}$ & $\begin{array}{l}\text { La diferencia entre los } \\
\text { mentirosos y los que } \\
\text { dicen la verdad será } \\
\text { mayor cuando los } \\
\text { entrevistados relaten sus } \\
\text { historias en orden } \\
\text { inverso, y la instrucción a } \\
\text { los entrevistados para que } \\
\text { recuerden sus historias en } \\
\text { orden inverso facilitará la } \\
\text { detección del engaño. }\end{array}$ \\
\hline 6 & $\begin{array}{l}\text { psycnet.a } \\
\text { pa.org }\end{array}$ & $\begin{array}{l}\text { Cook } \\
\text { al. }\end{array}$ & 2012 & $\begin{array}{l}\frac{\underline{\text { https:// }}}{\underline{\text { doi.org/ }}} \\
\frac{10.103}{\underline{7 / \mathrm{a} 002}} \\
\underline{8307}\end{array}$ & $\begin{array}{l}\text { Lyin' eyes: } \\
\text { Ocular- } \\
\text { motor } \\
\text { measures of } \\
\text { reading } \\
\underline{\text { reveal }} \\
\underline{\text { deception. }}\end{array}$ & $\begin{array}{l}\text { Evaluar una } \\
\text { alternativa a los } \\
\text { métodos actuales para } \\
\text { detectar el engaño en } \\
\text { contextos de control } \\
\text { de seguridad. }\end{array}$ & $\begin{array}{l}\text { Los participantes fueron } \\
\text { asignados al azar a un grupo } \\
\text { "culpable" o grupo "inocente" que } \\
\text { sólo se enteró del crimen. Luego } \\
\text { se presentaron para el ensayo, } \\
\text { donde rellenaron el cuestionario } \\
\text { administrado por computadora en } \\
\text { el que se abordaba su posible } \\
\text { participación en los delitos. }\end{array}$ & $\begin{array}{l}\text { Los presentes hallazgos } \\
\text { sugieren que hay dos } \\
\text { procesos cognitivos } \\
\text { implicados en el engaño - } \\
\text { vigilancia y estrategia- y } \\
\text { que estos procesos se } \\
\text { reflejan en diferentes } \\
\text { medidas } \\
\text { oculares }\end{array}$ & $\begin{array}{l}\text { Evaluamos una nueva } \\
\text { prueba de engaño basada } \\
\text { en la cognición que } \\
\text { medía las respuestas } \\
\text { ocular-motoras de los } \\
\text { participantes (respuestas } \\
\text { de los alumnos y } \\
\text { conductas de lectura) } \\
\text { mientras leían } \\
\text { respondían } \\
\text { declaraciones en un } \\
\text { cuestionario } \\
\text { computarizado. }\end{array}$ \\
\hline 7 & $\begin{array}{l}\text { psycnet.a } \\
\text { pa.org }\end{array}$ & $\begin{array}{l}\text { Lubow y } \\
\text { Fein. }\end{array}$ & 1996 & $\frac{\text { /doi.org }}{\frac{/ 10.103}{7 / 1076-}}$ & $\begin{array}{l}\text { Pupillary } \\
\text { size in } \\
\text { response to a }\end{array}$ & $\begin{array}{l}\text { Examinar los efectos } \\
\text { de cometer un delito } \\
\text { simulado en la }\end{array}$ & $\begin{array}{l}\text { Se registraron los electros } \\
\text { dérmicos y al momento de llevar } \\
\text { a cabo el interrogatorio a los }\end{array}$ & $\begin{array}{l}\text { Con los datos electro } \\
\text { dérmicos, el } 55 \% \text { de los } \\
\text { culpables y el } 93 \% \text { de los }\end{array}$ & $\begin{array}{l}\text { Los resultados son } \\
\text { prometedores para el } \\
\text { desarrollo de un nuevo }\end{array}$ \\
\hline
\end{tabular}




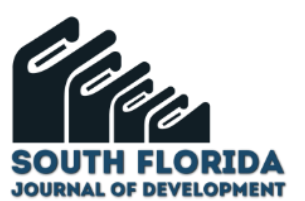

$\begin{array}{llllll}\frac{\text { 898X.2 }}{2.164} & \begin{array}{llll}\text { visual guilty } \\ \text { knowledge }\end{array} & \begin{array}{l}\text { respuesta pupilar (PR) } \\ \text { a las fotografías de los }\end{array} & \text { de culpabilidad. } & \text { fueron método de interrogación } \\ \text { test: New } & \text { detalles de la escena } & & \text { identificados } & \text { criminal usando PR a una } \\ & \underline{\text { technique for }} & \text { del crimen. } & \text { correctamente. } & \text { prueba de conocimiento } \\ \text { de culpabilidad visual. }\end{array}$

8 psycnet.a Berrien y 1943 https://

1943 https:// An

on.

$\underline{10.103}$

$\underline{7 / \mathrm{h} 006}$

exploratory

study

Identificar mentiras

Se realizó

mediante el estudio y

Se realizó

una entrevista

pupilometria.

la dilatación pupilar antes

respuestas engañosas

En

$48 \%$ de las

responses

during

verdaderas.

falsedades el sistem

vascular y la pupila dan

indicaciones de tensión

emocional. "Esto se

compara con el 18 por

ciento cuando se dan

respuestas veraces a

preguntas críticas."

Using reality

Mejorar la detección Ciento sesenta y seis estudiantes

Las declaraciones veraces

pa.org al.

$\underline{10.103}$

monitoring

del engaño con la fueron asignados aleatoriamente a

y engañosas difirieron

Los hallazgos apoyan el

7/lhb00 deception Realidad (RM: Vrij et Veracidad, jugaron un juego con

$\underline{00127} \underline{\text { detection in al., 2008), que ya ha un confederado, mientras que, en }}$ the context

significativamente

uso de los criterios de the sido validada en el

engaño en las entrevistas

cognitive

interview for testigos.

condión afectivos,

suspects.

participantes ensayaron una valic

sinopsis del escenario del juego. para la evaluación de la

Los participantes en la condición veracidad de la

Engañosa también fueron declaración $(\mathrm{p} \leq .01)$.

instruidos para robar 10 dólares Además, una regresión

de la cartera de un confederado. logística binaria encontró

En ambas condiciones, $\$ 10$ se que la combinación de los dieron por perdidos y un criterios de MR juntos investigador ciego a la condición clasificó correctamente el condujo un CIS. La veracidad de $86,6 \%$ de las la declaración fue codificada declaraciones, $\chi^{2}(6)=$ usando 6 de los criterios de RM 114,4, p <.001, con una avanzados por Vrij et al.

excelente sensibilidad $\mathrm{y}$ especificidad $(.899 \mathrm{y}$ .833 , respectivamente

10 www.tan Hugh \& 2009 https:// Selfdoi.org/ reported

Juzgar a dfonline. Young.

los Ciento cuatro estudiantes vieron una cinta de vídeo de seis

Los resultados indic com cues about veraces,

que los detectores de

Los detectores de que utilizaban 


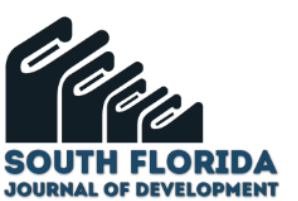

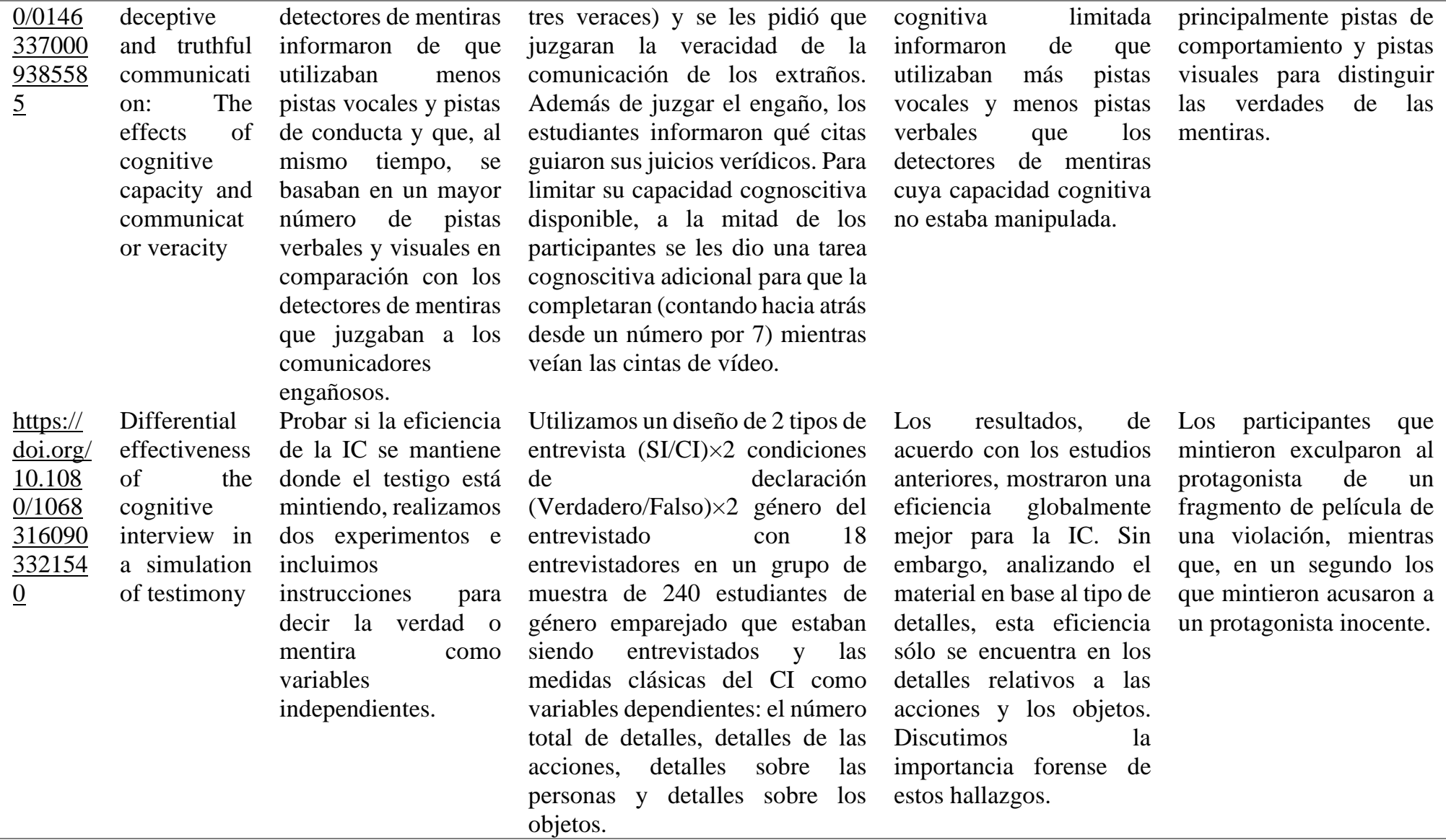




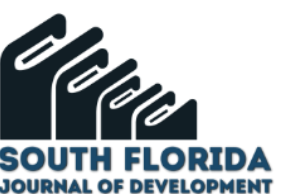

\begin{tabular}{|c|c|c|c|c|c|c|c|c|c|c|}
\hline 12 & $\begin{array}{l}\text { www.tan } \\
\text { dfonline. } \\
\text { com }\end{array}$ & $\begin{array}{l}\text { Man } \\
\text { Vrij. }\end{array}$ & $\mathrm{y}$ & 2007 & $\begin{array}{l}\frac{\frac{\text { https:// }}{\text { doi.org/ }}}{10.108} \\
\frac{0 / 1068}{316060} \\
\frac{055844}{4}\end{array}$ & $\begin{array}{l}\text { Police } \\
\text { officers' } \\
\text { judgements } \\
\text { of veracity, } \\
\text { tenseness, } \\
\text { cognitive } \\
\text { load and } \\
\text { attempted } \\
\text { behavioural } \\
\text { control in } \\
\text { real-life } \\
\text { police } \\
\text { interviews }\end{array}$ & $\begin{array}{l}\text { Examinar las } \\
\text { conclusiones } \\
\text { aparentemente } \\
\text { incongruentes de que } \\
\text { los agentes de policía } \\
\text { creen que los } \\
\text { sospechosos muestran } \\
\text { un comportamiento } \\
\text { nervioso cuando } \\
\text { mienten, pero parecen } \\
\text { capaces de detectar el } \\
\text { engaño en los } \\
\text { sospechosos que no } \\
\text { muestran } \\
\text { comportamientos } \\
\text { nerviosos. }\end{array}$ & $\begin{array}{l}\text { Los } 84 \text { policías vieron siete } \\
\text { verdades y siete mentiras dichas } \\
\text { por los sospechosos durante sus } \\
\text { entrevistas. Se pidió a los } \\
\text { participantes en la condición } 1 \\
\text { que indicaran después de cada } \\
\text { vídeo si el sospechoso mentía, } \\
\text { mientras que a los participantes en } \\
\text { la condición } 2 \text { se les pidió que } \\
\text { indicaran después de cada vídeo } \\
\text { hasta qué punto el sospechoso } \\
\text { parecía estar tenso, teniendo que } \\
\text { pensar mucho o tratando de } \\
\text { controlar su comportamiento. }\end{array}$ & $\begin{array}{l}\text { Los resultados apoyan las } \\
\text { hipótesis y por lo tanto } \\
\text { niegan la suposición de } \\
\text { que los agentes de policía } \\
\text { buscan principalmente } \\
\text { indicios de nerviosismo } \\
\text { cuando intentan detectar } \\
\text { un engaño. }\end{array}$ & $\begin{array}{l}\text { Se formuló la hipótesis de } \\
\text { que los juicios de los } \\
\text { agentes de policía sobre } \\
\text { si un sospechoso está } \\
\text { mintiendo no estarían } \\
\text { correlacionados con sus } \\
\text { juicios sobre si el } \\
\text { sospechoso está tenso, } \\
\text { sino que estarían } \\
\text { correlacionados con sus } \\
\text { juicios sobre si los } \\
\text { sospechosos tienen que } \\
\text { pensar mucho o intentan } \\
\text { controlar su } \\
\text { comportamiento. }\end{array}$ \\
\hline 13 & $\begin{array}{l}\text { www.tan } \\
\text { dfonline. } \\
\text { com }\end{array}$ & $\begin{array}{l}\text { Pfister } \\
\text { al. }\end{array}$ & et & 2014 & $\begin{array}{l}\frac{\text { https:// }}{\text { doi.org/ }} \\
\frac{10.108}{0 / 1747} \\
\frac{0919.2}{\underline{014.93}} \\
\underline{4392}\end{array}$ & $\begin{array}{l}\text { Pants on fire: } \\
\text { The } \\
\text { electrophysi } \\
\text { ological } \\
\text { signature of } \\
\text { telling a lie }\end{array}$ & $\begin{array}{l}\text { Identificar a la } \\
\text { electroencefalografía } \\
\text { como un papel } \\
\text { destacado en la } \\
\text { detección de mentiras }\end{array}$ & $\begin{array}{l}\text { Este diseño nos permitió estudiar } \\
\text { la firma de la mentira en ausencia } \\
\text { de raros y personalmente } \\
\text { significativos estímulos extraños } \\
\text { que se utilizan típicamente para la } \\
\text { detección de mentiras a través de } \\
\text { marcadores electrofisiológicos, } \\
\text { especialmente el componente } \\
\text { P300. }\end{array}$ & $\begin{array}{l}\text { Estos resultados apoyan } \\
\text { los relatos que enfatizan } \\
\text { la alta demanda cognitiva } \\
\text { de la mentira, incluyendo } \\
\text { la necesidad de suprimir } \\
\text { la respuesta verdadera y } \\
\text { generar una mentira. }\end{array}$ & $\begin{array}{l}\text { La electroencefalografía } \\
\text { ha desempeñado un papel } \\
\text { destacado en la detección } \\
\text { de mentiras a través de la } \\
\text { información } \\
\text { personalmente relevante, } \\
\text { la firma } \\
\text { electrofisiológica de la } \\
\text { mentira activa sigue } \\
\text { siendo esquiva. }\end{array}$ \\
\hline 14 & $\begin{array}{l}\text { www.tan } \\
\text { dfonline. } \\
\text { com }\end{array}$ & $\begin{array}{l}\text { Leal } \\
\text { Vrij. }\end{array}$ & $\mathrm{y}$ & 2010 & $\begin{array}{l}\frac{\frac{\text { https:// }}{\text { doi.org/ }}}{\frac{10.108}{0 / 1068}} \\
\frac{316090}{277684} \\
\underline{3}\end{array}$ & $\begin{array}{l}\text { The } \\
\text { occurrence } \\
\text { of eye blinks } \\
\text { during a } \\
\text { guilty } \\
\text { knowledge } \\
\text { test }\end{array}$ & $\begin{array}{l}\text { Examinar si el } \\
\text { parpadeo de los ojos } \\
\text { podría discriminar } \\
\text { entre los examinados } \\
\text { culpables e inocentes } \\
\text { en una Prueba de } \\
\text { Conocimiento } \\
\text { Culpable (GKT). }\end{array}$ & $\begin{array}{l}\text { Un total de } 26 \text { participantes } \\
\text { tomaron parte en un experimento } \\
\text { en el que } 13 \text { examinandos } \\
\text { culpables cometieron un robo } \\
\text { simulado (de un examen) y los } 13 \\
\text { examinandos inocentes restantes } \\
\text { continuaron con sus actividades } \\
\text { normales. }\end{array}$ & $\begin{array}{l}\text { Tanto los examinados } \\
\text { culpables como los } \\
\text { inocentes mostraron el } \\
\text { patrón de parpadeos que } \\
\text { predijimos. }\end{array}$ & $\begin{array}{l}\text { Formulamos la hipótesis } \\
\text { de que mostrarían menos } \\
\text { parpadeos durante los } \\
\text { elementos clave que } \\
\text { durante los elementos de } \\
\text { control. }\end{array}$ \\
\hline 15 & $\begin{array}{l}\text { www.tan } \\
\text { dfonline. } \\
\text { com }\end{array}$ & $\begin{array}{l}\text { Frank } \\
\text { al. }\end{array}$ & et & 2018 & $\begin{array}{l}\frac{\text { https:// }}{\text { doi.org/ }} \\
\underline{\underline{10.108}} \\
\underline{0 / 0269} \\
\underline{9931.2}\end{array}$ & $\begin{array}{l}\text { The } \\
\text { language of } \\
\text { lies: a } \\
\text { preregistered } \\
\text { direct }\end{array}$ & $\begin{array}{lr}\text { Encontrar } & \text { las } \\
\text { diferencias } & \text { de } \\
\text { mentira-verdad en las } \\
\text { RT en } & \text { los } \\
\text { participantes al } & \text { ser }\end{array}$ & $\begin{array}{l}\text { Midieron el tiempo que los } \\
\text { hablantes nativos de alemán } \\
\text { necesitaban para iniciar } \\
\text { respuestas honestas y engañosas a } \\
\text { preguntas en alemán e inglés. }\end{array}$ & $\begin{array}{l}\text { Estos hallazgos indican } \\
\text { que las personas luchan } \\
\text { por recuperar } \\
\text { rápidamente la verdad en } \\
\text { otro idioma, y que el uso }\end{array}$ & $\begin{array}{l}\text { La hipótesis de la } \\
\text { distancia emocional y la } \\
\text { carga cognitiva dan } \\
\text { respuestas que compiten } \\
\text { entre sí. }\end{array}$ \\
\hline
\end{tabular}




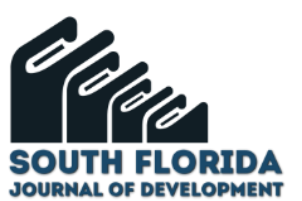

\begin{tabular}{|c|c|c|c|c|c|c|c|c|c|}
\hline & & & & $\underline{018.15}$ & $\begin{array}{l}\text { replication } \\
\text { of Suchotzki } \\
\text { and Gamer } \\
(2018 ; \\
\text { Experiment } \\
\text { 2) }\end{array}$ & $\begin{array}{l}\text { evaluados en inglés y } \\
\text { al ser evaluados en } \\
\text { holandés. }\end{array}$ & & $\begin{array}{l}\text { de un idioma extranjero } \\
\text { puede disminuir las } \\
\text { diferencias entre las } \\
\text { mentiras y las verdades. }\end{array}$ & \\
\hline 16 & $\begin{array}{l}\text { www.tan } \\
\text { dfonline. } \\
\text { com }\end{array}$ & $\begin{array}{l}\text { Bembibr } \\
\text { e \& \& } \\
\text { Higueras. }\end{array}$ & 2012 & $\begin{array}{l}\frac{\frac{\text { https:// }}{\text { doi.org/ }}}{\frac{10.108}{0 / 1068}} \\
\frac{316 X .2}{\frac{011.58}{9387}}\end{array}$ & $\begin{array}{l}\text { Comparative } \\
\text { analysis of } \\
\text { true or false } \\
\text { statements } \\
\text { with the } \\
\text { source } \\
\text { monitoring } \\
\text { model and } \\
\text { the cognitive } \\
\text { interview: } \\
\text { special features of } \\
\text { the false } \\
\text { accusation of } \\
\text { innocent } \\
\text { people }\end{array}$ & $\begin{array}{l}\text { Verificar si las } \\
\text { categorías del modelo } \\
\text { de monitoreo de la } \\
\text { fuente (SM) y la } \\
\text { entrevista cognitiva } \\
\text { (CI) contribuyen a } \\
\text { distinguir las } \\
\text { declaraciones } \\
\text { verdaderas de las } \\
\text { falsas. }\end{array}$ & $\begin{array}{l}18 \text { entrevistadores en un grupo de } \\
\text { muestra de } 240 \text { estudiantes } \\
\text { universitarios de sexo equivalente } \\
\text { que fueron entrevistados sobre un } \\
\text { fragmento de película, los } \\
\text { participantes que mintieron } \\
\text { exculparon al protagonista de la } \\
\text { violación, mientras Que las } \\
\text { mentiras estaban dirigidas a } \\
\text { acusar a una persona inocente. }\end{array}$ & $\begin{array}{l}\text { Los resultados sugieren } \\
\text { que las declaraciones de } \\
\text { los participantes honestos } \\
\text { entrevistados utilizando } \\
\text { la IC proporcionaron más } \\
\text { información general que } \\
\text { las obtenidas bajo } \\
\text { cualquier otra condición. }\end{array}$ & $\begin{array}{l}\text { Modelo de monitoreo de } \\
\text { la fuente (SM) y la } \\
\text { entrevista cognitiva (CI) } \\
\text { contribuyen a distinguir } \\
\text { las declaraciones } \\
\text { verdaderas de las falsas. }\end{array}$ \\
\hline 17 & $\begin{array}{l}\text { www.tan } \\
\text { dfonline. } \\
\text { com }\end{array}$ & $\begin{array}{l}\text { Vernham } \\
\text { \& Vrij. }\end{array}$ & 2015 & $\begin{array}{l}\frac{\frac{\text { https:// }}{\text { doi.org/ }}}{10.108} \\
\frac{0 / 2374}{4006.2} \\
\underline{015.10} \\
\underline{51756}\end{array}$ & $\begin{array}{l}\text { A review of } \\
\text { the } \\
\text { collective } \\
\text { interviewing } \\
\text { approach to } \\
\text { detecting } \\
\text { deception in } \\
\text { pairs }\end{array}$ & $\begin{array}{l}\text { Recapitular la } \\
\text { importancia de la } \\
\text { detección de mentiras } \\
\text { y de cuándo se pueden } \\
\text { utilizar las entrevistas } \\
\text { colectivas, antes de } \\
\text { resumir los estudios } \\
\text { sobre el engaño en las } \\
\text { entrevistas colectivas } \\
\text { publicados hasta la } \\
\text { fecha. }\end{array}$ & $\begin{array}{l}\text { Se realizo comparaciones entre } \\
\text { las entrevistas individuales y } \\
\text { colectivas con el mensaje para } \\
\text { llevar a casa de que las entrevistas } \\
\text { colectivas no deben sustituir a las } \\
\text { individuales, sino que deben } \\
\text { utilizarse ambos tipos de } \\
\text { entrevistas, tal vez a veces } \\
\text { conjuntamente. }\end{array}$ & $\begin{array}{l}\text { Los estudios publicados } \\
\text { muestran que la falta de } \\
\text { señales interactivas y } \\
\text { comunicativas, como } \\
\text { formularse preguntas, } \\
\text { corregirse, interrumpirse, } \\
\text { terminar las frases y } \\
\text { mirarse, son indicadores } \\
\text { significativos de engaño. }\end{array}$ & $\begin{array}{l}\text { En el examen se destaca } \\
\text { que las teorías sobre la } \\
\text { memoria y la dinámica de } \\
\text { grupo son cruciales para } \\
\text { comprender el engaño } \\
\text { que se produce dentro de } \\
\text { los grupos y, por lo tanto, } \\
\text { deberían ser el centro de } \\
\text { futuros estudios sobre el } \\
\text { engaño en las entrevistas } \\
\text { colectivas. }\end{array}$ \\
\hline 18 & $\begin{array}{l}\text { www.tan } \\
\text { dfonline. } \\
\text { com }\end{array}$ & $\begin{array}{l}\text { Dukala et } \\
\text { al. }\end{array}$ & 2008 & $\begin{array}{l}\frac{\frac{\text { https:// }}{\text { doi.org } /}}{10.130} \\
\underline{\underline{0 / J 070 \mathrm{v}}} \\
\underline{\underline{08 \mathrm{n} 01}} \\
\underline{06}\end{array}$ & 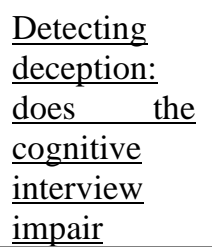 & $\begin{array}{l}\text { Explicar cómo es } \\
\text { probable que estas } \\
\text { distorsiones se } \\
\text { desarrollen a partir de } \\
\text { los procesos } \\
\text { cognitivos normales }\end{array}$ & $\begin{array}{l}\text { La metodología que se utiliza } \\
\text { dentro de esta investigación es de } \\
\text { estructura descriptiva. }\end{array}$ & $\begin{array}{l}\text { Se proporciona un marco } \\
\text { que distingue entre dos } \\
\text { grandes grupos de } \\
\text { autoengaño sobre la base } \\
\text { del conjunto de funciones }\end{array}$ & $\begin{array}{lr}\text { El concepto } & \text { de } \\
\text { autoengaño motivado } \\
\text { para explicar cómo es } \\
\text { probable que estas } \\
\text { distorsiones } & \text { se } \\
\text { desarrollen a partir de los }\end{array}$ \\
\hline
\end{tabular}




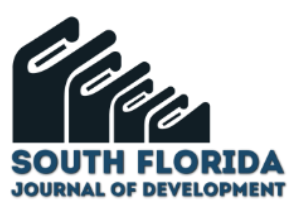

\section{discriminati}

discir

on

criteria in

elderly

witnesses?

19 www.tan Cardona 2013 https://

dfonline. \& doi.org

com Quevedo.

$\frac{\text { doi.org/ }}{10.310}$

$\frac{10.310}{9 / 0271}$

3683.2

$\underline{013.84}$

$\underline{1256}$

Blinking and Investigar

Driving: the

Influence of

$\underline{\text { Saccades }}$

and

$\underline{\text { Cognitive }}$

Workload

www.els

evier.es

Blandón

et al.

2017

10.101

6/j.apj.

2017.0

2.004

Cognition,

emotion, and

lying:

Implications

to detect

deception

21 www.els Terola et

evier.es

al.

10.101

6/j.apj.

2014.0

6.004

Detection of concealed

information using event-

related

potentials

Argumentar

mentir no es siempre

más complejo que

decir la verdad.

cognitivas y las

sacadas de gran

amplitud en la tasa de

parpadeo espontáneo. cuándo una persona miente y cuándo dice la verdad desde un punto de vista forense circ

de conducción de 60

minutos en la vida real mientras

una cámara de vídeo grababa los

movimientos oculares y el

parpadeo. Las sacadas se

clasificaron según su amplitud y
se registraron pares de sacadas de

parpadeo.

Es un trabajo de una revisión

sistemática de artículos.

(a)

Hallar métodos que Se pretende hacer una revisión de nos ayuden a saber los artículos que estudian estos procedimientos

distintos métodos: propiedades, fiabilidad, validez y limitaciones.

Confirmar o negar ncbi.nlm. et al. nih.gov

$2012 \quad \underline{10.137}$ $\underline{1 / \text { journ }}$

al.pone.

004025

$\underline{9}$
The eyes Confirmar o negar
don't have it: que los postulados de

lie detection la PNL.

and Neuro-

Linguistic

part

Los movimientos oculares de los

participantes que estaban

estaban codificados. En otro

grupo de participantes fue intrín

procesos

normales.

cognitivos

En los diferentes niveles En un entorno visual de complejidad, no se complejo y dinámico, el encontró ninguna parpadeo espontáneo que diferencia acompaña a las sacadas estadísticamente de gran amplitud puede significativa en la tasa de ayudar a contrarrestar la parpadeo espontáneo, con reducción de la tasa de un promedio de $20,3 \pm$ parpadeo asociada a las 1,6 parpadeos/minuto altas exigencias (media $\pm \mathrm{SD})$. cognitivas.

Inferir automáticamente Ésta sostiene que mentir engaño a partir de es cognitivamente más indicadores de carga complejo que decir la cognitiva puede llevar a verdad y que un aumento error puesto que las en la dificultad cognitiva emociones influyen en la durante una entrevista cognición, no puede hará que el mentiroso obviarse su papel en la muestre señales delatoras detección de mentiras.

e sobrecarga cognitiva.

Los resultados indican La posibilidad de tasas de acierto en la "falsear" las respuestas discriminación de llevan a cuestionar la culpables en un rango de inexactitud utilizada en 7 al 100 por ciento, y en algunos círculos la de inocentes de 31 a mediáticos respecto a las 100 por ciento.

cualidades y finalidades de dicha prueba.

En conjunto, los Los defensores de la resultados de los tres Programación Neuroestudios no apoyan las Lingüística (PNL) afirmaciones de la PNL. afirman que ciertos movimientos oculares 


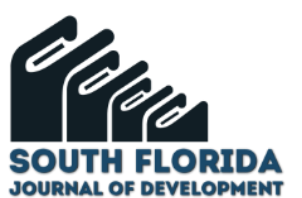

Programmin

23 ncbi.nlm. et al. nih.gov

nih.gov

pubmed. Curci et $2018-10.338$

ncbi.nlm. al.

nih.gov $\frac{9 / \text { fpsyt }}{2018.0}$

\section{$\mathrm{g}$}

lie detection

by inducing

cognitive

load on liars:

a review of

relevant

theories and

techniques

guided by

lessons from

polygraph-

based

approaches.

Measuring

9/fpsyg

.2015 .0

$\underline{0596}$

the cognitive

resources

consumed

per second

for real-time

lie-

production

and

recollection:

a dual-

tasking

paradigm

Accuracy,

Confidence,

and

Experiential

Criteria for

Lie

Detection

Through
Presentar un método

novedoso

detección de mentiras

de doble tarea.

críticamente

as técnicas y teorías

relevantes para el

campo emergente de

la "detección de

una carga cognitiva

electiva en los exitosa.

mentirosos".

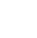

los movimientos oculares de la

PNL mientras que un segundo grupo de control no lo fue.

Comenzamos con un resumen de la Técnica de Preguntas Controladas (CQT) basada en polígrafos y las principales críticas. También se consideran las lecciones de la prueba de conocimiento culpable más 


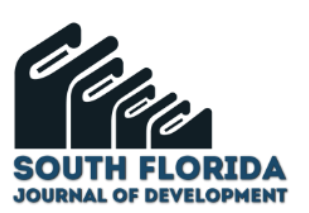

Videotaped

Interview

cuenta la complejidad

cognitiva y la escasez de

manifestaciones

expresivas relacionadas con el informe del entrevistado.

26 pubmed. Bockstae

ncbi.nlm. le et al.

nih.gov

ncbi.nlm. et al.

nih.gov $\underline{10.338}$ 9 fpsyt. 2 $\underline{018.00}$ $\underline{700}$
A Review of Approaches to Detecting Malingering in Forensic Contexts and Promising

Cognitive

Load-

Inducing Lie

Detection

Techniques

www.tan Jeffrey et $2016 \quad 10.108$ More Than

dfonline. al

com
$0 / 0742$

1222.2

016.12

05929
Meets the

Eye: How

Oculometric

Behaviors

Evolve Over

the Course of

Automated

Deception
Explorar más a fondo En el estudio $(\mathrm{n}=42)$, en una los efectos de la prueba de mentira de Sheffield en práctica sobre la tres fases: Línea de base (50\% capacidad de mentir

mentira,

$50 \%$

verdad),

de una persona Entrenamiento (grupo de mentiras manipulando las frecuentes: $75 \%$ mentira, $25 \%$ proporciones de la verdad; grupo de control: $50 \%$ mentira y las pruebas mentira, 50\% verdad; y grupo de de verdad.

verdades frecuentes: 25\% mentira, $75 \%$ verdad), y Prueb (50\% mentira, $50 \%$ verdad).

Detectar si los Se revisan los métodos más pacientes fingen una comunes de detección de dolencia psicológica o física para lucrar.

simulacros, incluidos aquellos para fingir deficiencias psiquiátricas y cognitivas. El artículo continúa con una discusión sobre los medios de uso común para detectar el engaño.

Lamentablemente, sus tasas de falsos positivos y falsos negativos tienden a ser altas. síntomas y como detectar la verdad en pacientes que fijen enfermedades.

\begin{tabular}{lllr} 
Los resultados indican & Las & \multicolumn{2}{r}{ investigaciones } \\
que los indicadores & anteriores & sobre el \\
oculométricos de los & engaño & mediante \\
engañadores evolucionan & rastreadores oculares se \\
de manera diferente en el & han centrado en la & la \\
curso de una interacción, & detección & e \\
y que estas tendencias son & interpretación de breves \\
indicativas de engaño & variaciones & \\
independientemente de & $\begin{array}{l}\text { oculométricas } \\
\text { respuesta a estímulos }\end{array}$
\end{tabular}

Los resultados indican Las investigaciones evolucionan cómo Utilizando el modelo de la curva las de crecimiento latente, probamos variaciones en los hipótesis que explican cómo dos comportamientos comportamientos oculométricos oculométricos en el pupilas dilatadas y patrones de curso de una fijación de la mirada.

interacción con un

sistema de detección de engaños.
Las teorías cognitivas que mentir requiere más recursos cognitivos que decir la verdad. no persiste a 10 de la mentira, aunque este 


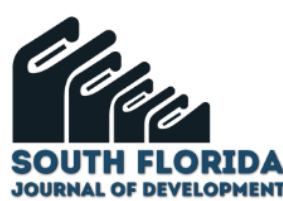

Detection

Interactions

se muestren (por ejemplo, imágenes

estímulos pertinentes. específicas o preguntas

de entrevistas).

29 www.sci Nurçina encedirec et al. t.com encedirec al. t.com

www.sci Cho y 2018 encedirec lee. t.com www.sci Choi et 2016 encedirec al t.com

33 www.sci Heaver \& 2010 encedirec Hutton. t.com $\underline{\text { https:// }}$

doi.org

$\underline{10.101}$

6/j.proc

$\frac{\text { s. } 2017 .}{11.258}$

https://

doi.org/

10.101

$\underline{6 / \mathrm{B} 978}$

-0-12-

$=$

$\overline{7.0000}$

$\underline{5-1}$

https:// Comparison

doi:10. of Cognitive

1016/j.i Load and

jpsycho Emotional

.2018.0 Arousal on

7.214 Liar's Pupil

Diameter

Changes

dis

Demostrar que la Mediante de la utilización del Los sirve como indicador del engaño. https:// A Possibility Examinar

doi.org/ of Pupil posibilidad de

$\underline{10.101}$ Diameter as

6/j.ijps Reliable

ycho.20 Index for Lie $\underline{16.07 .4}$ Detection. 35

10.101 Keeping an Detectar las mentiras 6/j.ijps eye on the en las personas que ycho. 20 truth: Pupil

\section{programa de culpabilidad} la pupila como inocentes realizaron las diferentes indicador de la misiones que fueron diseñadas detección

de para robar.

$$
\text { Utilizamos la }
$$

Todas las fotos tomadas Entrenamiento de un del iris se ingresaron software para la correctamente a la red detección de la mentira a neuronal para el través de la medición de entrenamiento en la la dilatación de la pupila. detección de mentiras.

El uso de la respuesta Realizar una propuesto pupilar y los de enfoque innovador movimientos oculares para detectar el engaño durante la lectura que utiliza la respuesta promete como una nueva pupilar. herramienta para la detección del engaño.

Los

mentirosos El presente estudio $\begin{array}{lrl}\text { experimentan } & \text { una } & \text { sugiere que inducir } \\ \text { excitación } & \text { emocional } & \text { La carga cognitiva es más }\end{array}$ debido a la ansiedad o a la efectiva que la inducción sensación de culpa, pero también están emocional. influenciados por la carga cognitiva ya que la mentira es más exigente cognitivamente que decir la verdad.

Estos hallazgos indicaron dilatación pupilar de servir como ser detectado a través de $\begin{array}{ll}\text { indicador dentro de las entrevistas } \\ \text { dentono }\end{array}$ los cambios en el de tipo de pregunta. diámetro de la pupila. en video
cambios 


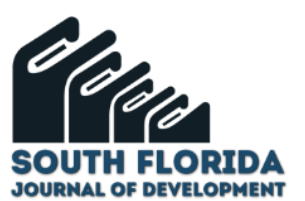

10.06.2 size, fingen enfermedad en la pupila durante una prueba de

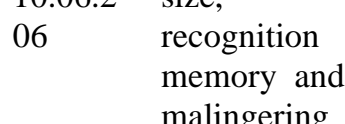

34

www.sci Lee \& 2018

encedirec Lee.

t.com

www.sci Fisher

encedirec

t.com

16/j.iss of Eye malingering

fingen enfermedad
con un fin económico.

$10.101 \quad$ Increase

6/j.ijps Lie

ycho.20 Detection

18.07.3 Accuracy

36 through

Integrated

Measuremen

$\mathrm{t}$ of Pupil

Polygraph

2010 https://

doi.org/ lies, you can

$\frac{10.101}{6 / \mathrm{S} 026}$

$\underline{6 / \mathrm{S} 026}$

2-

$4079(1$

$\underline{0) 6085}$

9-7 tell by its

eyes

$$
\begin{array}{ll}
\text { eyes } & \text { indicado } \\
& \text { engaño- }
\end{array}
$$

Dilation and of Verificar si es más efectivo reemplazar el polígrafo con el índice de la pupila utilizarlo

integralmente con el polígrafo.

Determinar si los Los investigadores preguntaron a movimientos de los 11 voluntarios sobre preguntas jos serven como personales,

del y les dijo que mintieran en algún de sus respuestas. Durante el

\section{memoria de reconocimiento cuando}

10.121 Application Investigar el valor de

$\underline{\text { n.1004- }}$ Tracker in seguimiento ocular en

$\underline{5619.2}$ Lie Detection mentiras. cuando los participantes

se les dio instrucciones de memoria de reconocimiento estándar, instrucciones para

fingir amnesia e instrucciones para reportar todos los artículos como nuevos. entrevistas, los voluntarios llevaban gafas de rastreo de ojos que grabaron

su velocidad de parpadeo, dirección y

la longitud de la mirada, y la movimiento de los ojos dilatación de la pupila. Los 40 sujetos se

ven los artículos viejos

comparados con los

nuevos artículos replica

los anteriores

investigación

documenta el efecto

antiguo/nuevo de la

pupila.

Los resultados sugieren que no sólo el índice de la pupila complementa las

investigado si el índice de la pupila es un sustituto o complemento del polígrafo

polígrafos, sino también el uso complementario de los polígrafos y

de las pupilas aumenta la precisión de la detección de mentiras.

En promedio, los Movimientos de los ojos, participantes $88 \%$

de las verdades correctamente cuando los avatares

tenía movimiento ocular, pero sólo 70\%, la más difícil, pero el ayudó. leatoriamente en dos grupos, en grupo experimental pupila, la duración media l grupo experimental se pretende tenían un diámetro de de la fijación y los puntos observar la estimulación del pupila promedio más de fijación obtenidos por objetivo y se registraron con el corto, una duración el seguidor ocular en 


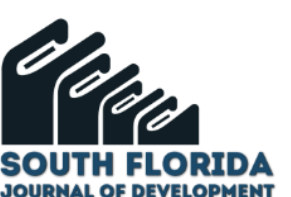

\section{$\underline{015}$}

pubmed. Taniguch 2013

ncbi.nlm. i \& Ono.

nih.gov

38 dl.acm.or Iqbal et 2004

$\mathrm{g}$ al.
020.02. $\quad$ rastreador ocular después de que lograron el crimen simulado. promedio de fijación más

larga y menos puntos de fijación $(P<0,05)$,

pero las diferencias en la

frecuencia de parpadeo

no tuvieron significación

estadística

$\begin{array}{lll}\text { Non-contact } & \text { Se investigar la } \\ \text { measuremen } & \text { eficacia de la }\end{array}$

Se utilizó un protocolo de Los resultados

no Los movimientos de los

mostraron diferencias ojos como indicadores

significativas en los del engaño, según los

movimientos oculares resultados de los estudios

entre las imágenes se observa que es posible

críticas y no críticas en la detectar el engaño

condición de alta mediante los

similitud. movimientos de los ojos.

in the de los movimientos imagen crítica. Los participantes deception] contacto.

de alta o baja similitud. Se les presentaron al azar imágenes críticas y no críticas y se midieron sus movimientos oculares sin contacto.

10.114 Task evoked Evaluar de manera un usuario realizó una tarea más $\underline{5 / 9859}$ pupil precisa la carga de fácil y más difícil

response to trabajo mental de un de varias categorías de tareas y mental para

workload in desarrollar sistemas

humande atención

computer usuario.

del medimos el tamaño de la pupila de la pupila mediante un interaction.

rastreador ocular montado en la calificaciones mayores cabeza. Utilizamos el tiempo de de tiempo de finalización de la tarea y las valoraciones subjetivas de la dificultad para

validar la carga mental impuesta por las tareas de mayor respuesta

pupilar en

correspondientes
Los resultados muestran Creemos que

que una tarea más difícil el tamaño de la pupila es exige más tiempo de

tiempo de procesamiento, prometedora de la

mayores mental porque no

de trabajo mental y evoca usuario

de forma fiable una subtareas que una tarea menos

difícil. de usuario, y es menos intrusivo que otras

medidas fisiológicas como la frecuencia cardíaca o el EEG. de trabajo mental del 